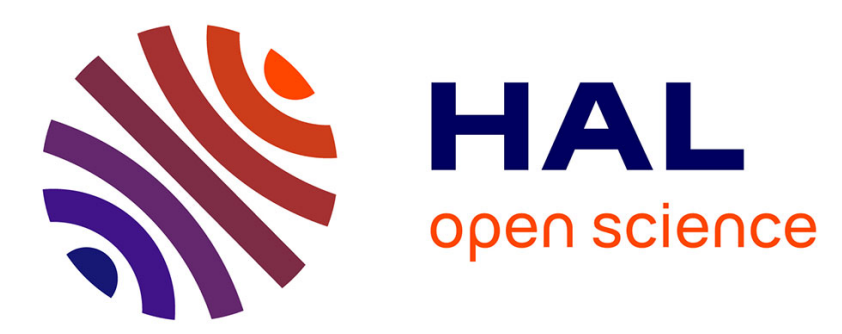

\title{
A seamless hybrid RANS-LES model based on transport equations for the subgrid stresses and elliptic blending
}

Atabak Fadai-Ghotbi, Christophe Friess, Remi Manceau, Jacques Borée

\section{To cite this version:}

Atabak Fadai-Ghotbi, Christophe Friess, Remi Manceau, Jacques Borée. A seamless hybrid RANSLES model based on transport equations for the subgrid stresses and elliptic blending. Physics of Fluids, 2010, 22 (5), pp.055104. 10.1063/1.3415254 . hal-02132038

\section{HAL Id: hal-02132038 \\ https://hal.science/hal-02132038}

Submitted on 16 May 2019

HAL is a multi-disciplinary open access archive for the deposit and dissemination of scientific research documents, whether they are published or not. The documents may come from teaching and research institutions in France or abroad, or from public or private research centers.
L'archive ouverte pluridisciplinaire HAL, est destinée au dépôt et à la diffusion de documents scientifiques de niveau recherche, publiés ou non, émanant des établissements d'enseignement et de recherche français ou étrangers, des laboratoires publics ou privés. 


\title{
A seamless hybrid RANS-LES model based on transport equations for the subgrid stresses and elliptic blending
}

\author{
Atabak Fadai-Ghotbi, ${ }^{\text {a) }}$ Christophe Friess, Rémi Manceau, ${ }^{\text {b) }}$ and Jacques Borée \\ Department of Fluid Flow, Heat Transfer and Combustion, Institute Pprime, CNRS, \\ University of Poitiers, ENSMA, SP2MI, Bd. Marie et Pierre Curie, BP 30179, \\ 86962 Futuroscope Chasseneuil Cedex, France
}

(Received 2 November 2009; accepted 24 March 2010; published online 7 May 2010)

\begin{abstract}
The aim of the present work is to develop a seamless hybrid Reynolds-averaged Navier-Stokes (RANS) large-eddy simulation (LES) model based on transport equations for the subgrid stresses, using the elliptic-blending method to account for the nonlocal kinematic blocking effect of the wall. It is shown that the elliptic relaxation strategy of Durbin is valid in a RANS (steady) as well as a LES context (unsteady). In order to reproduce the complex production and redistribution mechanisms when the cutoff wavenumber is located in the productive zone of the turbulent energy spectrum, the model is based on transport equations for the subgrid-stress tensor. The partially integrated transport model (PITM) methodology offers a consistent theoretical framework for such a model, enabling to control the cutoff wavenumber $\kappa_{c}$, and thus the transition from RANS to LES, by making the $C_{\varepsilon_{2}}$ coefficient in the dissipation equation of a RANS model a function of $\kappa_{c}$. The equivalence between the PITM and the Smagorinsky model is shown when $\kappa_{c}$ is in the inertial range of the energy spectrum. The extension of the underlying RANS model used in the present work, the elliptic-blending Reynolds-stress model, to the hybrid RANS-LES context, brings out some modeling issues. The different modeling possibilities are compared in a channel flow at $\operatorname{Re}_{\tau}=395$. Finally, a dynamic procedure is proposed in order to adjust during the computation the dissipation rate necessary to drive the model toward the expected amount of resolved energy. The final model gives very encouraging results in comparison to the direct numerical simulation data. In particular, the turbulence anisotropy in the near-wall region is satisfactorily reproduced. The contribution of the resolved and modeled fields to the Reynolds stresses behaves as expected: the modeled part is dominant in the near-wall zones (RANS mode) and decreases toward the center of the channel, where the relative contribution of the resolved part increases. Moreover, when the mesh is modified, the amount of resolved energy changes but the total Reynolds stresses remain nearly constant.
\end{abstract}

(C) 2010 American Institute of Physics. [doi:10.1063/1.3415254]

\section{INTRODUCTION}

Problems ranging from noise prediction to fluid/structure interaction or thermal fatigue require the computation of time-dependent characteristics of complex flows. Reynoldsaveraged Navier-Stokes (RANS) computations are often used in industrial configurations because of their low computational cost, which is weakly dependent on the Reynolds number, but are not able to provide unsteady information. On the contrary, large-eddy simulation (LES) can provide the necessary information by resolving the large-scale structures and modeling the smaller scales, which have a more universal behavior. However, at high Reynolds numbers, the computational cost of LES is too high for complex industrial applications. One reason is that the cutoff wavenumber, separating resolved and modeled scales, must be sufficiently large for the energetic scales to be resolved, leading to the use of fine meshes. In particular, a limitation of LES is the resolution required for the crucial near-wall region, which is

\footnotetext{
${ }^{a)}$ Present address: SNECMA, Site de Villaroche, Rond-point René RavaudRéau, 77550 Moissy-Cramayel, France.

b) Author to whom correspondence should be addressed. Electronic mail: remi.manceau@univ-poitiers.fr.
}

to be solved in a quasi-direct numerical simulation (DNS) mode, in order to avoid the use of wall functions.

Therefore, a wide variety of relatively low-cost strategies (compared to LES) have recently emerged for performing unsteady computations: ${ }^{1}$ very large-eddy simulation (VLES), ${ }^{2}$ limited numerical scales (LNS), ${ }^{3}$ detached eddy simulation (DES), ${ }^{4}$ unsteady RANS (URANS), ${ }^{5-9}$ organized eddy simulation (OES), ${ }^{10}$ scale-adaptive simulation (SAS), ${ }^{11}$ partially averaged Navier-Stokes, ${ }^{12}$ partially integrated transport model (PITM), ${ }^{13,14}$ and additive RANS/DNS filtering, ${ }^{15-17}$ among others.

Computations based on a RANS model in some regions of the flow, in particular in the near-wall zones and on LES in some other regions, where explicit computation of the large-scale structures is required, as in separated zones, are referred to as hybrid RANS-LES computations. When the transition RANS-LES occurs in a continuous manner, the model is said to be seamless or continuous, sometimes global $^{1}$ or unified. ${ }^{18}$ In homogeneous flows, this type of model can be seen as a LES with a cutoff wavenumber continuously going to zero or, equivalently, as a LES with a filter width continuously going to infinity, this limit corresponding formally to the RANS approach (spatial average). For inho- 
mogeneous and stationary flows, similar approaches can be formulated in the framework of temporal filtering, leading to hybrid temporal LES-RANS models. ${ }^{19,20}$

Using such a model between a RANS region and a LES region, there is necessarily, by continuity, a region where the cutoff wavenumber is located in the energetic part of the spectrum. The challenge is thus to be able to reproduce the complex production and redistribution mechanisms occurring at these scales, which are very difficult, if not impossible at all, to be accounted for using an algebraic relation between the subgrid-stress tensor and the resolved strain tensor. In the context of unsteady RANS, it has been highlighted by several authors, in the case of a turbulent field subject to an imposed, periodic strain (e.g., Hadžić, Hanjalić, and Laurence; ${ }^{21}$ Carpy and Manceau ${ }^{22}$ ), that a second-moment closure is necessary to provide a correct physical representation. Thus, in the present paper, a model based on transport equations for the subgrid-stress tensor is developed. This approach was initially suggested in the early work of Deardorff, ${ }^{23}$ and followed, e.g., by Schumann ${ }^{24}$ and more recently by Chaouat and Schiestel $^{14}$ and Perot and Gadebusch. $^{25}$ The better representation of the physical mechanisms is at the price of a moderate increase in the computational cost, due to the additional transport equations. However, a model that is able to correctly reproduce the physical mechanisms when the cutoff wavenumber is in the energetic range is expected to be able to run in VLES mode far from the wall, i.e., on meshes too coarse to perform a standard LES. A slight coarsening of the mesh can by far compensate for the cost of solving additional transport equations.

Another challenge is to provide a theoretical framework to the separation resolved/modeled scales which bridges RANS and LES. Recently, such a theoretical framework has been proposed, ${ }^{13}$ the so-called PITM, and used with transport equations for the subgrid-stress tensor and the dissipation rate. ${ }^{14}$ As a result of modeling in spectral space, with a variable cutoff wavenumber $\kappa_{c}$, compatibility is guaranteed with RANS $\left(\kappa_{c} \rightarrow 0\right)$ and LES.

The originality of the present work is the use of transport equations for the subgrid-scale (SGS) stresses based on the application of the elliptic-blending strategy to reproduce the nonviscous, nonlocal blocking effect of the wall. Similar to the RANS context, ${ }^{26}$ elliptic relaxation is a method based on a theoretical argumentation that enables the reproduction of the influence of the wall on turbulence without the introduction of so-called "damping function," which is empirical and lacks theoretical foundations, in particular for unsteady velocity and pressure fields. The present model is thus an adaptation to the hybrid RANS-LES approach of the ellipticblending Reynolds-stress model (EB-RSM), ${ }^{27,28}$ which is a near-wall extension of the Speziale-Sarkar-Gatski (SSG) model, ${ }^{29}$ using the elliptic relaxation strategy of Durbin. ${ }^{26}$ This model was successfully applied to different configurations in a RANS framework. ${ }^{28,30-36}$

The aim of the present paper is thus to adapt the ellipticblending model to the hybrid context, using the PITM methodology. ${ }^{13,14}$ Section II introduces the general filtering approach of Germano, ${ }^{37}$ applied to the Navier-Stokes equations, which provides an unified formalism for RANS and LES equations. The model equations of the PITM and the elliptic-blending strategy are presented in Sec. III. The adaptation of the EB-RSM brings out some issues which are exposed in Sec. III C. The numerical methods are provided in Sec. IV A, and then the modeling issues are investigated and discussed in Secs. IV B-IV D. Finally, in Sec. V, a dynamic procedure is proposed to adapt the dissipation rate in order to reach the expected balance between resolved and modeled energy. A channel flow at $\operatorname{Re}_{\tau}=395$ is used to evaluate the modeling options, in comparison against DNS data. ${ }^{38}$

\section{FILTERED EQUATIONS}

As usual in LES, the formalism is introduced in the frame of homogeneous flows, and extended to inhomogeneous flows afterwards. The instantaneous velocity and pressure fields are denoted by $U_{i}^{*}$ and $P^{*}$, respectively. The instantaneous flow is driven by the incompressible NavierStokes equations

$$
\begin{aligned}
& \frac{\partial U_{i}^{*}}{\partial t}+U_{j}^{*} \frac{\partial U_{i}^{*}}{\partial x_{j}}=-\frac{1}{\rho} \frac{\partial P^{*}}{\partial x_{i}}+\nu \frac{\partial^{2} U_{i}^{*}}{\partial x_{j} \partial x_{j}}, \\
& \frac{\partial U_{j}^{*}}{\partial x_{j}}=0 .
\end{aligned}
$$

In the context of LES and hybrid methods, the instantaneous velocity is decomposed into a resolved part $\widetilde{U}_{i}$ and a residual part $u_{i}^{\prime \prime}$, such that

$$
U_{i}^{*}=\tilde{U}_{i}+u_{i}^{\prime \prime} .
$$

The resolved velocity is obtained by the application to the instantaneous velocity of a filter $\mathcal{F}$, of characteristic width $\Delta_{f}$

$$
\tilde{U}_{i}(\mathbf{x}, t)=\left\langle U_{i}^{*}\right\rangle=\int \mathcal{F}(\mathbf{x}, \boldsymbol{\xi}) U_{i}^{*}(\boldsymbol{\xi}, t) \mathrm{d} \boldsymbol{\xi},
$$

where the brackets denote the filtering operator. The most common filters are the Gaussian filter, the top-hat filter, and the spectral cutoff filter. In the case of a spectral cutoff filter, the cutoff wavenumber separating resolved and modeled scales is defined by $\kappa_{c}=\pi / \Delta_{f}$. Wavenumbers smaller than $\kappa_{c}$ in the turbulent energy spectrum are explicitly resolved, whereas wavenumbers higher than $\kappa_{c}$ are modeled (see Fig. 1). The ensemble average - (Reynolds average) of $U_{i}^{*}$ is denoted by $U_{i}=\overline{U_{i}^{*}}$. The large-scale fluctuation is defined by

$$
u_{i}^{\prime}=\tilde{U}_{i}-U_{i},
$$

and the total fluctuation by

$$
u_{i}=U_{i}^{*}-U_{i}=u_{i}^{\prime}+u_{i}^{\prime \prime} .
$$

Similarly, the instantaneous pressure $P^{*}$ is decomposed into a filtered, resolved part $\widetilde{\mathcal{P}}$ and a residual fluctuating part $p^{\prime \prime}$. The filtered field is incompressible, i.e., $\partial_{k} \widetilde{U}_{k}=0$, and the filtered momentum equation is written as ${ }^{37}$ 


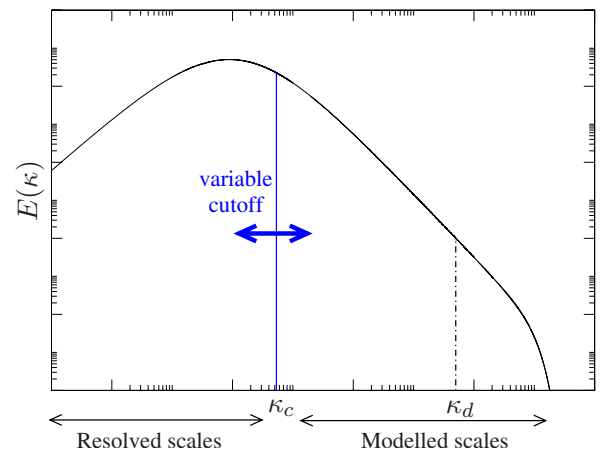

FIG. 1. (Color online) Definition of the wavenumbers $\kappa_{c}$ and $\kappa_{d}$.

$$
\frac{\tilde{D} \tilde{U}_{i}}{\tilde{D} t}=-\frac{1}{\rho} \frac{\partial \widetilde{\mathcal{P}}}{\partial x_{i}}+\nu \frac{\partial^{2} \widetilde{U}_{i}}{\partial x_{j} \partial x_{j}}-\frac{\partial \tau_{i j}}{\partial x_{j}},
$$

where $\tilde{D} / \tilde{D} t=\partial_{t}+\tilde{U}_{k} \partial_{k}$ and $\tau_{i j}$ is the SGS tensor, which is the tensor of the generalized central moments $\tau_{i j}=\tau\left(U_{i}^{*}, U_{j}^{*}\right)$ defined by $\tau(a, b)=\langle a b\rangle-\langle a\rangle\langle b\rangle$ for any variables $a$ and $b$. The exact transport equation for $\tau_{i j}$ is given by Germano ${ }^{37}$

$$
\begin{aligned}
& \frac{\tilde{D} \tau_{i j}}{\tilde{D} t}=\underbrace{-\frac{\partial \tau\left(U_{i}^{*}, U_{j}^{*}, U_{k}^{*}\right)}{\partial x_{k}}}_{D_{i j_{\mathrm{SGS}}^{T}}}+\underbrace{\nu \frac{\partial^{2} \tau_{i j}}{\partial x_{k} \partial x_{k}}}_{D_{i j_{\mathrm{SGS}}}^{\nu}}-\underbrace{2 \nu \tau\left(\frac{\partial U_{i}^{*}}{\partial x_{k}}, \frac{\partial U_{j}^{*}}{\partial x_{k}}\right)}_{\varepsilon_{i j_{\mathrm{SGS}}}}
\end{aligned}
$$

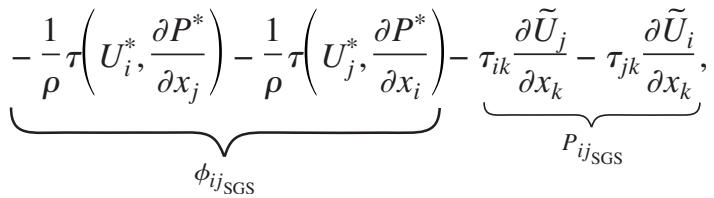

where

$$
\begin{aligned}
\tau(a, b, c)= & \langle a b c\rangle-\langle a\rangle \tau(b, c)-\langle b\rangle \tau(a, c)-\langle c\rangle \tau(a, b) \\
& -\langle a\rangle\langle b\rangle\langle c\rangle .
\end{aligned}
$$

$P_{i j_{\mathrm{SGS}}}, \varepsilon_{i j_{\mathrm{SGS}}}, D_{i j_{\mathrm{SGS}}}^{\nu}, D_{i j_{\mathrm{SGS}}}^{T}$, and $\phi_{i j_{\mathrm{SGS}}}$ represent SGS production, dissipation, viscous diffusion, turbulent diffusion, and velocity-pressure gradient correlation, respectively. Hereafter, $\phi_{i j_{\mathrm{SGS}}}$ is called the pressure term for convenience.

In homogeneous flows, one of the advantages of using the generalized central moments is that for any filter defined by Eq. (4), the total Reynolds stress $R_{i j}=\overline{u_{i} u_{j}}$ can be simply decomposed as ${ }^{37}$

$$
R_{i j}=\left(\overline{\tilde{U}_{i} \widetilde{U}_{j}}-U_{i} U_{j}\right)+\overline{\tau_{i j}} .
$$

On the right hand side of Eq. (10), $\overline{\tau_{i j}}$ is the modeled part of the Reynolds stress, and the term in parentheses represents the resolved part. The total fluctuating kinetic energy is defined by $k=\frac{1}{2} R_{i i}=k_{m}+k_{r}$, where the modeled part is

$$
k_{m}=\overline{k_{\mathrm{SGS}}}=\frac{1}{2} \overline{\tau_{i i}}
$$

and the resolved part

$$
k_{r}=\frac{1}{2}\left(\overline{\tilde{U}_{i} \tilde{U}_{i}}-U_{i} U_{i}\right)=\frac{1}{2} \overline{u_{i}^{\prime} u_{i}^{\prime}}
$$

A noteworthy feature of Eq. (8) is that it is of the same form as the standard RANS equations. When the cutoff wavenumber goes to zero, such that all the turbulent scales are modeled, $\tau_{i j} \rightarrow R_{i j}, k_{r} \rightarrow 0$, and $k_{m} \rightarrow k$. In this case, the RANS limit is formally reached in homogeneous flows, and the filtered field corresponds to a spatially averaged field, equivalent to the ensemble-averaged field. ${ }^{19}$ This feature provides the basis for consistently bridging RANS and LES methods.

\section{EQUATIONS OF THE MODEL}

In this section, the hybrid RANS-LES approach, to socalled PITM, initially proposed by Schiestel and Dejoan, ${ }^{13}$ is first introduced (for a detailed presentation of all the steps of the derivation of this approach, the reader is referred to the original articles $\left.{ }^{13,14,39}\right)$. Then, the compatibility of the method with Smagorinsky-like models is analyzed in the case of a cutoff wavenumber in the inertial range. In this consistent framework, the SGS transport equations are modeled and, in particular, the accounting of wall effects by elliptic blending is proposed.

\section{A. Hybrid RANS-LES approach: The PITM 1. Rationale and equations}

In seamless hybrid RANS/LES methods, the amount of resolved energy is to be controlled by making the equations of the model dependent on the filter width. As shown by Schiestel and Dejoan, ${ }^{13}$ this can be achieved by using a transport equation for the dissipation rate that is a modification of the usual equation used in RANS. In order to know how to modify this equation in order to make it dependent on the cutoff wavenumber, homogeneous turbulence is considered, and the turbulence spectrum is partitioned into three regions, $\left[0, \kappa_{c}\right],\left[\kappa_{c}, \kappa_{d}\right]$, and $\left[\kappa_{d}, \infty\right]$, such that the model is a particular case, reduced to only three spectral zones of the multiscale models proposed by Schiestel. ${ }^{40-42} \kappa_{c}$ is the cutoff wavenumber, characterizing the filter introduced in Sec. II. The interval $\left[0, \kappa_{c}\right]$ is explicitly resolved, whereas the interval $\left[\kappa_{c}, \infty\right]$ is to be modeled. The wavenumber $\kappa_{d}$ is defined by the relation

$$
\kappa_{d}=\kappa_{c}+\zeta_{m} \frac{\varepsilon}{k_{m}^{3 / 2}},
$$

where $\varepsilon=\overline{\varepsilon_{\mathrm{SGS}}}=\frac{1}{2} \overline{\varepsilon_{j j_{\mathrm{SGS}}}}$ is the Reynolds-averaged dissipation rate and the constant $\zeta_{m}$ is chosen sufficiently large, such that the contribution of the zone $\left[\kappa_{d}, \infty\right]$ to the total energy is negligible (see Fig. 1). This also implies that the energy transfer at $\kappa_{d}$ can be assumed equal to the dissipation rate, i.e., spectral equilibrium is satisfied at this scale. ${ }^{13}$

In homogeneous flows, the evolution equation of the energy spectrum reads 


$$
\frac{\partial E}{\partial t}=-\lambda_{i j} \mathcal{A}_{i j}+\mathcal{T}-2 \nu \kappa^{2} E,
$$

where $\lambda_{i j}$ is the mean velocity gradient tensor, $\mathcal{A}_{i j}$ the spectral tensor integrated over a spherical shell, and $\mathcal{T}$ the spectral transfer term. Integrating Eq. (14) in the two ranges of wavenumbers $\left[\kappa_{c}, \infty\right]$ and $\left[\kappa_{d}, \infty\right]$, introducing them into Eq. (13) and taking the time derivative, it can be shown, ${ }^{13}$ after some algebraic manipulations, that the derivative of the subgrid turbulent energy and the dissipation rate can be written in a form similar to the usual RANS equations

$$
\frac{d k_{m}}{d t}=P_{m}-\varepsilon
$$

and

$$
\frac{d \varepsilon}{d t}=C_{\varepsilon_{1}} \frac{P_{m} \varepsilon}{k_{m}}-\underbrace{\left(C_{\varepsilon_{1}}-\frac{k_{m}}{\varepsilon} \frac{\frac{d \kappa_{d}}{d t}-\frac{d \kappa_{c}}{d t}}{\kappa_{d}-\kappa_{c}}\right)}_{C_{\varepsilon_{2}}^{*}} \frac{\varepsilon^{2}}{k_{m}},
$$

where $P_{m}=\overline{P_{\mathrm{SGS}}}=\frac{1}{2} \overline{P_{j j_{\mathrm{SGS}}}}$ is the production rate of SGS energy. Although these equations are formally similar to the standard RANS equations, they involve SGS rather than Reynolds-averaged variables. In order to guarantee compatibility with the RANS limit, $C_{\varepsilon_{2}}^{*}$ must be equal to the RANS coefficient $C_{\varepsilon_{2}}$ when $\kappa_{c}=0$ and $d \kappa_{c} / d t=0$. This constraint leads to

$$
\frac{d \kappa_{d}}{d t}=\left(C_{\varepsilon_{1}}-C_{\varepsilon_{2}}\right) \frac{\varepsilon}{k} \kappa_{d} .
$$

Introducing Eq. (17) into the value of $C_{\varepsilon_{2}}^{*}$ defined in Eq. (16), and using the fact that $\kappa_{c} \ll \kappa_{d}$, the coefficient $C_{\varepsilon_{2}}^{*}$ is found implicitly dependent on the filter width through the relation

$$
C_{\varepsilon_{2}}^{*}=C_{\varepsilon_{1}}+r\left(C_{\varepsilon_{2}}-C_{\varepsilon_{1}}\right) .
$$

$r=k_{m} / k$ is the ratio modeled energy over total energy, which is dependent on the cutoff wavenumber. As proposed by Schiestel and Dejoan, ${ }^{13}$ using a spectral cutoff filter and a Kolmogorov energy spectrum, the ratio $r$ can be linked to the cutoff wavenumber $\kappa_{c}$ by

$$
\begin{aligned}
r & =\frac{1}{k} \int_{\kappa_{c}}^{\infty} E(\kappa) \mathrm{d} \kappa=\frac{1}{k} \int_{\kappa_{c}}^{\infty} C_{K} \varepsilon^{2 / 3} \kappa^{-5 / 3} \mathrm{~d} \kappa \\
& =\frac{3 C_{K}}{2}\left(\kappa_{c} \frac{k^{3 / 2}}{\varepsilon}\right)^{-2 / 3} .
\end{aligned}
$$

It is worth pointing out that in the present section, the study of the way to modify the dissipation equation is carried out using Fourier transforms in homogeneous flows. Consequently, Eq. (16) involves averaged quantities $\varepsilon, k_{m}$, and $P_{m}$. It is assumed that the relations between averaged quantities obtained in spectral space can be applied to filtered quantities. Therefore, Eq. (16) will be solved for the filtered dissipation rate $\varepsilon_{\mathrm{SGS}}=\frac{1}{2} \varepsilon_{j j_{\mathrm{SGS}}}$, sensitized to the cutoff wavenumber by Eq. (18). This practice corresponds to assuming that the level of SGS energy $k_{\mathrm{SGS}}$ will fluctuate around the average value $k_{m}$, which is controlled by the variable $C_{\varepsilon_{2}}^{*}$ coefficient. However, since the relations (16) and (18) are true only for averaged quantities, transposing them to resolved, fluctuating quantities does not ensure that the expected ratio $r$ of resolved energy over total energy will be reached in the simulations. This issue will be addressed in Sec. V. Moreover, the flows considered being inhomogeneous, molecular and turbulent diffusion terms will be introduced in the equations.

\section{Compatibility with the Smagorinsky model}

In the present approach, contrary to standard LES, the characteristic length scale of subgrid turbulence is not directly related to the grid, but evaluated using the dissipation rate given by Eqs. (16) and (18). This equation can be associated with an eddy-viscosity model, ${ }^{13}$ as well as a secondmoment closure. ${ }^{14}$ The parameter $r$ controls the transition from a RANS to a LES behavior, the RANS equation being recovered when $r=1$. When $r$ is reduced, typically around the value 0.2 , the cutoff wavenumber is well in the inertial range of the energy spectrum. The aim of this section is to show the compatibility of the hybrid approach with standard LES in this case.

If spectral equilibrium is assumed, a Kolmogorov spectrum can be used

$$
E(\kappa)=C_{K} \varepsilon^{2 / 3} \kappa^{-5 / 3},
$$

with $C_{K} \simeq 1.5$. The SGS kinetic energy defined by Eq. (11) then reads

$$
k_{m}=\int_{\kappa_{c}}^{\infty} E(\kappa) \mathrm{d} \kappa=\frac{3 C_{K}}{2} \varepsilon^{2 / 3} \kappa_{c}^{-2 / 3} .
$$

If, as usual in LES, the cutoff wavenumber is related to the mesh size $\Delta$ by $\kappa_{c}=\pi / \Delta$, Eq. (21) leads to

$$
\frac{k_{m}^{3 / 2}}{\varepsilon}=\left(\frac{3 C_{K}}{2}\right)^{3 / 2} \frac{\Delta}{\pi} \simeq 1.07 \Delta,
$$

which shows that in case of spectral equilibrium, the length scale of subgrid turbulence is directly linked to the mesh size, similarly to LES. However, this is only a particular case, and the PITM does not assume spectral equilibrium at the cutoff wavenumber $\kappa_{c}$, but only at wavenumber $\kappa_{d}$, as mentioned in Sec. III A 1. When the mesh is locally coarse compared to a LES mesh, the cutoff wavenumber can be located in the productive zone of the spectrum, where equilibrium is rarely achieved, and in such a case, determining the length scale through transport equations for dissipation and SGS energy (or SGS stresses) is crucial.

In the inertial range of the spectrum, second-moment closures and eddy-viscosity models for the SGSs are equivalent, due to the equilibrium and the isotropy pertaining to these scales, such that the following analysis is restricted to an eddy-viscosity model, for simplicity. The subgrid viscosity is written as 


$$
\nu_{t}=C_{\mu} \frac{k_{\mathrm{SGS}}^{2}}{\varepsilon_{\mathrm{SGS}}},
$$

where $C_{\mu}$ is a constant to be determined. Local equilibrium and Boussinesq relation imply

$$
\varepsilon=\overline{\varepsilon_{\mathrm{SGS}}}=C_{\mu} \frac{\overline{k_{\mathrm{SGS}}^{2}} \widetilde{S}^{2},}{\varepsilon_{\mathrm{SGS}}}
$$

with $\widetilde{S}_{i j}=1 / 2\left(\partial_{j} \widetilde{U}_{i}+\partial_{i} \widetilde{U}_{j}\right)$ and $\widetilde{S}=\sqrt{2 \widetilde{S}_{i j} \widetilde{S}_{i j}}$. Writing

$$
\overline{\frac{k_{\mathrm{SGS}}^{2}}{\varepsilon_{\mathrm{SGS}}} \widetilde{S}^{2}}=\beta \frac{k_{m}^{2}}{\varepsilon} \overline{\widetilde{S}^{2}},
$$

where $\beta$ is a correlation coefficient, and combining Eq. (24) with Eq. (21) leads to

$$
\varepsilon=\beta^{3 / 2} C_{\mu}^{3 / 2}\left(\frac{3 C_{K}}{2}\right)^{3}\left(\frac{\Delta}{\pi}\right)^{2}{\widetilde{S^{2}}}^{3 / 2} .
$$

Now, the Smagorinsky model yields

$$
\varepsilon=\left(C_{s} \Delta\right)^{2} \overline{\widetilde{S}^{3}},
$$

where the usual evaluation ${ }^{43}$ of the Smagorinsky constant is

$$
C_{s}=\frac{1}{\pi}\left(\frac{2}{3 C_{K}}\right)^{3 / 4}\left(\frac{{\overline{\widetilde{S}^{2}}}^{3 / 2}}{\overline{\widetilde{S}^{3}}}\right)^{1 / 2} .
$$

Compatibility between Eqs. (26) and (27) then requires

$$
C_{\mu}=\frac{1}{\beta}\left(\frac{2}{3 C_{K}}\right)^{3}
$$

Since $\left(2 / 3 C_{K}\right)^{3} \simeq 0.088$, if the correlation coefficient $\beta$ is assumed to be close to unity, the compatibility with the Smagorinsky model is ensured if the standard RANS value $C_{\mu}$ $\simeq 0.09$ is chosen. It is worth pointing out that if the correlation coefficient $\beta$ is lower than unity, the coefficient $C_{\mu}$ is to be increased for cutoff wavenumbers in the inertial regions, which is against intuition.

This result shows that the PITM, which is obviously compatible with standard RANS models when the cutoff wavenumber is equal to zero $\left(r=1, k_{m}=k\right)$, is also compatible with standard LES, when the cutoff wavenumber is in the inertial range. This is consistent with previous numerical studies showing the behavior of the PITM in the case of decay of isotropic turbulence, both using subgrid-viscosity models ${ }^{13}$ or, very recently, subgrid-stress models. ${ }^{44}$

When the model is neither in RANS mode nor in LES mode, i.e., when the cutoff wavenumber is in the productive region of the spectrum (VLES mode), the situation is different. The SGSs are subject to large-scale strain variations, and in such a case, the eddy-viscosity models fail and, in particular, the phase shift between stress and strain must be accounted for. ${ }^{22,45}$ Several authors ${ }^{10,46}$ showed that reducing the value of $C_{\mu}$ is beneficial in this case, but such models are not compatible with the LES limit, which cannot be reached by reducing $C_{\mu}$, as shown by the analysis above. The alteration of the length scales linked to the location of the cutoff wavenumber must be accounted for by another mechanism, which can be a modification of the dissipation rate in the subgrid energy equation (DES) or of the transport equation for the dissipation rate (PITM).

\section{B. Elliptic-blending model in the hybrid context}

In case of a wall-bounded LES at very high Reynolds number, the near-wall regions must be solved in a quasi-DNS mode, leading to a dramatic increase in the computational cost. One of the aims of the hybrid methods is to solve these regions in a RANS mode, which is cheaper and weakly dependent on the Reynolds number. A second aim of the hybrid methods is to allow relatively coarse meshes, meaning that the cutoff wavenumber can be in the productive range of the spectrum. In this range, important complex physical processes must be taken into account, such as production and redistribution. A second-moment closure provides a much better representation of these physical processes, in comparison to eddy-viscosity models, as can be shown by studying the interaction of a turbulent background with a time-varying strain. ${ }^{21,22}$ In Eq. (8), subgrid-stress production $P_{i j_{\mathrm{SGS}}}$ and viscous diffusion $D_{i j_{\mathrm{SGS}}}^{\nu}$ do not require modeling, and models will be discussed for the pressure $\phi_{i j_{\mathrm{SGS}}}$, dissipation $\varepsilon_{i j_{\mathrm{SGS}}}$, and turbulent diffusion $D_{i j_{\mathrm{SGS}}}^{T}$ terms.

\section{Pressure term}

The most crucial term to be modeled in Eq. (8) is the pressure term $\phi_{i j_{\mathrm{SGS}}}$, which is at the origin of the redistribution mechanisms and the nonlocal blocking effect due to the wall. The latter effect can be taken into account in singlepoint RANS closures using the elliptic relaxation strategy of Durbin, ${ }^{26,47}$ or simplified formulations such as the EB-RSM, ${ }^{27,28}$ which has been successfully applied in many flows, ${ }^{28,30-36}$ in a RANS context. An adaptation of the EBRSM is used here in the hybrid context for several reasons. First, unlike classical near-wall models, the EB-RSM does not make use of damping functions, which are arbitrary and, in principle, not valid for fluctuating quantities. On the contrary, it will be shown in the present section that the elliptic relaxation approach and, consequently, its simplified form, the elliptic-blending approach, are valid for filtered quantities, since they are derived from the Poisson equation for pressure fluctuations and asymptotic behaviors in the vicinity of the wall, which are formally identical in the RANS (statistical averaging) and the hybrid (filtering) formulations. Therefore, the main objective of the present work, compared to existing hybrid models based on transport equations to account for SGSs, ${ }^{13,14,25}$ is to avoid the use of damping functions in the near-wall region by introducing the nonlocal effects through elliptic blending. Finally, the elliptic-blending model is numerically robust, contrary to other models based on elliptic relaxation, for which the boundary conditions lead to strong numerical instabilities, and it is also less expansive since a single additional equation is to be solved, compared to the six additional equations of the original elliptic relaxation method of Durbin. ${ }^{26}$ In the present section, the validity of the elliptic relaxation strategy for filtered quantities is first demonstrated. Then, the simplified formulation, the so-called elliptic-blending approach, is adapted to the hybrid context. 
a. Elliptic relaxation strategy in the hybrid context. Subtracting the filtered equation (7) from the instantaneous equation (1), one can deduce the evolution equation for the subgrid fluctuating field

$$
\begin{aligned}
\frac{\partial u_{i}^{\prime \prime}}{\partial t}+u_{j}^{\prime \prime} \frac{\partial u_{i}^{\prime \prime}}{\partial x_{j}}= & -\frac{1}{\rho} \frac{\partial p^{\prime \prime}}{\partial x_{i}}+\nu \frac{\partial^{2} u_{i}^{\prime \prime}}{\partial x_{j} \partial x_{j}} \\
& +\frac{\partial}{\partial x_{j}}\left(\tau_{i j}-\tilde{U}_{i} u_{j}^{\prime \prime}-\tilde{U}_{j} u_{i}^{\prime \prime}\right),
\end{aligned}
$$

with the kinematic incompressibility constraint $\partial_{j} u_{j}^{\prime \prime}=0$. Taking the divergence of Eq. (30), it is deduced that the residual fluctuating part of the pressure, and therefore its gradient, satisfy a Poisson equation

$$
\nabla^{2} \frac{\partial p^{\prime \prime}}{\partial x_{k}}=\underbrace{\rho \frac{\partial}{\partial x_{k}}\left[-2 \frac{\partial \tilde{U}_{i}}{\partial x_{j}} \frac{\partial u_{j}^{\prime \prime}}{\partial x_{i}}-\frac{\partial^{2}}{\partial x_{i} \partial x_{j}}\left(u_{i}^{\prime \prime} u_{j}^{\prime \prime}-\tau_{i j}\right)\right]}_{\mathcal{S}_{k}} .
$$

Using Green's formalism, ${ }^{48,49}$ the solution to Eq. (31) is given by

$$
\frac{\partial p^{\prime \prime}}{\partial x_{k}}(\mathbf{x})=\int \frac{\mathcal{S}_{k}(\mathbf{x}+\mathbf{r})}{4 \pi\|\mathbf{r}\|} \mathrm{d} \mathbf{r},
$$

where $1 /(4 \pi\|\mathbf{r}\|)$ is Green's function associated with the operator $\nabla^{2}$. The time dependence of the variables is not explicitly written in order to simplify the notation. As shown by Eq. (32), the subgrid pressure gradient is nonlocal since it results of a spatial integration. Assuming a spectral cutoff filter to simplify the formalism, the pressure term, defined in Eq. (8), can be written as

$$
\rho \phi_{i j_{\mathrm{SGS}}}=-\left\langle u_{i}^{\prime \prime} \frac{\partial p^{\prime \prime}}{\partial x_{j}}\right\rangle-\left\langle u_{j}^{\prime \prime} \frac{\partial p^{\prime \prime}}{\partial x_{i}}\right\rangle .
$$

Note that the following results can be extended to any filter. Using Eq. (32), the integral equation of $\phi_{i j_{\mathrm{SGS}}}$ reads

$$
\rho \phi_{i j_{\mathrm{SGS}}}(\mathbf{x})=-\int_{\mathcal{D}} \frac{\left\langle u_{i}^{\prime \prime}(\mathbf{x}) \mathcal{S}_{j}(\mathbf{x}+\mathbf{r})+u_{j}^{\prime \prime}(\mathbf{x}) \mathcal{S}_{i}(\mathbf{x}+\mathbf{r})\right\rangle}{4 \pi\|\mathbf{r}\|} \mathrm{d} \mathbf{r} .
$$

Similar to the elliptic relaxation model applied in the RANS context, $^{47}$ a simple shape is assumed for the two-point correlation, of the form

$$
\begin{aligned}
& \left\langle u_{i}^{\prime \prime}(\mathbf{x}) \mathcal{S}_{j}(\mathbf{x}+\mathbf{r})+u_{j}^{\prime \prime}(\mathbf{x}) \mathcal{S}_{i}(\mathbf{x}+\mathbf{r})\right\rangle \\
& \quad=\left\langle u_{i}^{\prime \prime}(\mathbf{x}+\mathbf{r}) \mathcal{S}_{j}(\mathbf{x}+\mathbf{r})+u_{j}^{\prime \prime}(\mathbf{x}+\mathbf{r}) \mathcal{S}_{i}(\mathbf{x}+\mathbf{r})\right\rangle \exp \left(-\frac{\|\mathbf{r}\|}{L_{\mathrm{SGS}}}\right),
\end{aligned}
$$

where $L_{\mathrm{SGS}}$ is a correlation length scale. The main difference between Eq. (35) and the one found in RANS lies in the fact that the former is written for filtered variables, while the latter is written for Reynolds-averaged variables. Consequently, the length scale, which was modeled as a function of Reynolds-averaged quantities in RANS, must in the present case be modeled as a function of SGS quantities, and as such, will be dependent on the cutoff wavenumber. This modeling issue will be investigated in Sec. IV.

The use of an isotropic form of the correlation function, with the same length scale for all the directions, is a strong assumption. The validity of this approximation was investigated, by extracting the correlation function from a DNS database, by Manceau et l. $^{48}$ in the frame of RANS modeling. It was shown that the correlation function is indeed strongly anisotropic in the near-wall region, but also that the main consequence of the isotropic assumption, the underestimation of wall-blocking in the logarithmic layer, is due to the asymmetry of the correlation function in the wall-normal direction. The elongation in the tangential directions does not affect the redistribution process, due to statistical homogeneity, and, consequently, it was shown that the length scale entering the modeled, isotropic correlation function must be calibrated in order to correctly reproduce the nonlocal effects in the wall-normal direction. Furthermore, several modifications of the elliptic relaxation model were proposed to enhance the wall-blocking in the logarithmic layer, ${ }^{48,50-52}$ but the elliptic-blending approach, ${ }^{27}$ a simplification of elliptic relaxation, introduced in Sec. III B 1 b, does not exhibit this weakness.

In the frame of hybrid RANS/LES, the elongation of the correlation function in the directions tangent to the wall cannot be rigorously neglected, since the redistribution term is not constant in these directions anymore. However, since the hybrid formulation presented in the subsequent sections enforces the RANS mode in the near-wall region, the gradients in the tangential directions remain weak and the main effect to be reproduced is still the nonlocality in the wall-normal direction. The unique length scale entering Eq. (35) will be carefully calibrated in Sec. IV C to reproduce this effect. Since the length scale of the correlation function in Eq. (34) is shorter in the wall-normal direction than in the other directions, ${ }^{48}$ this procedure can, however, lead to an underestimation of the nonlocal effect in the tangential directions.

Using the model equation (35), the integral equation for $\phi_{i j_{\mathrm{SGS}}}$ becomes

$$
\begin{aligned}
\rho \phi_{i j_{\mathrm{SGS}}}(\mathbf{x})= & -\int_{\mathcal{D}} \underbrace{\left\langle u_{i}^{\prime \prime}(\mathbf{x}+\mathbf{r}) \mathcal{S}_{j}(\mathbf{x}+\mathbf{r})+u_{j}^{\prime \prime}(\mathbf{x}+\mathbf{r}) \mathcal{S}_{i}(\mathbf{x}+\mathbf{r})\right\rangle}_{\Psi_{i j}(\mathbf{x}+\mathbf{r})} \\
& \times \frac{\exp \left(-\frac{\|\mathbf{r}\|}{L_{\mathrm{SGS}}}\right)}{4 \pi\|\mathbf{r}\|} \mathrm{d} \mathbf{r} .
\end{aligned}
$$

The function $\exp \left(-\|\mathbf{r}\| / L_{\mathrm{SGS}}\right) /(4 \pi\|\mathbf{r}\|)$ being Green's function associated with the operator $\nabla^{2}-1 / L_{\mathrm{SGS}}^{2}$, Eq. (36) is the solution of the so-called elliptic relaxation equation

$$
\phi_{i j_{\mathrm{SGS}}}-L_{\mathrm{SGS}}^{2} \nabla^{2} \phi_{i j_{\mathrm{SGS}}}=\frac{L_{\mathrm{SGS}}^{2}}{\rho} \Psi_{i j}
$$

Again, this result shows that the RANS (Reynolds-averaged) and hybrid (filtered) versions of the elliptic relaxation model are formally identical, although they are written for different variables. Following the common practice in RANS, the 
right hand side of Eq. (37) can be modeled using a local model $\phi_{i j}^{h}$, i.e., a model that does not account for the nonlocality of the pressure term presented above, such that

$$
\phi_{i j_{\mathrm{SGS}}}-L_{\mathrm{SGS}}^{2} \nabla^{2} \phi_{i j_{\mathrm{SGS}}}=\phi_{i j}^{h} .
$$

The particular model used for $\phi_{i j}^{h}$ will be detailed in Sec. III B $1 \mathrm{~b}$, dedicated to the elliptic-blending model, which is a simplified formulation of the elliptic relaxation model.

$b$. Elliptic-blending model in the hybrid context. As noted by Manceau and Hanjalic ${ }^{27}$ in RANS, the system (38) is somewhat redundant since it applies the same operator, with the same correlation length scale, to all the components

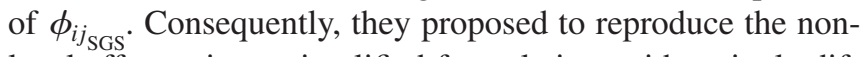
local effect using a simplified formulation, with a single differential equation instead of six. First, the elliptic relaxation equation is solved for a blending coefficient $\alpha$

$$
\alpha-L_{\mathrm{SGS}}^{2} \nabla^{2} \alpha=1 .
$$

The elliptic-blending strategy then consists in blending the "homogeneous" (away from the wall) and the near-wall models of the pressure term $\phi_{i j_{\mathrm{SGS}}}$ using

$$
\phi_{i j_{\mathrm{SGS}}}=(1-f) \phi_{i j}^{w}+f \phi_{i j}^{h} .
$$

Originally, Manceau and Hanjalić ${ }^{27}$ used $f=k \alpha$ as the blending function in this equation, but Manceau ${ }^{28}$ showed that using a power of $\alpha$ is equivalent close to the wall and avoids erroneous behaviors in several configurations. Therefore, $f$ $=\alpha^{3}$ is used in the present work. The boundary condition of Eq. (39) at the wall is simply $\alpha=0$, such that $\alpha$ goes from zero at the wall, to unity far from the wall. This parameter is an implicit indicator of the distance to the wall. The choice of the correlation length scale $L_{\mathrm{SGS}}$ is crucial and will be discussed in Sec. IV. In the RANS context, Manceau and Hanjalic $^{27}$ showed that the appropriate form of $\phi_{i j}^{w}$ can be obtained by an analysis of the asymptotic behaviors at the wall, in order to satisfy the balance between the pressure, molecular diffusion, and dissipation terms in the vicinity of the wall. Since in the present approach, the near-wall zone will be treated in RANS mode, the near-wall form $\phi_{i j}^{w}$ used in RANS does not require modification to be used in the hybrid model. It will appear in the results below that the near-wall region is never fully steady, since it is subject to large-scale oscillations coming from the outer region, but the analysis of Manceau and Hanjalić ${ }^{27}$ already accounted for this possibility. Therefore, the same form as in RANS can be used

$$
\phi_{i j}^{w}=-5 \frac{\varepsilon_{\mathrm{SGS}}}{k_{\mathrm{SGS}}}\left[\tau_{i k} n_{j} n_{k}+\tau_{j k} n_{i} n_{k}-\frac{1}{2} \tau_{k l} n_{k} n_{l}\left(n_{i} n_{j}+\delta_{i j}\right)\right],
$$

where $\mathbf{n}=\boldsymbol{\nabla} \alpha /\|\nabla \alpha\|$ is a generalized wall-normal vector.

Due to the formal equivalence of RANS and LES motion equations (see Sec. II), the SSG model of Speziale et al., ${ }^{29}$ usually applied in a RANS context, is assumed to be applicable here for $\phi_{i j}^{h}$

$$
\begin{aligned}
\phi_{i j}^{h}= & -\left(g_{1}+g_{1}^{*} \frac{P_{\mathrm{SGS}}}{\varepsilon_{\mathrm{SGS}}}\right) \varepsilon_{\mathrm{SGS}} b_{i j}+\left(g_{3}-g_{3}^{*} \sqrt{b_{k l} b_{k l}}\right) k_{\mathrm{SGS}} \tilde{S}_{i j} \\
& +g_{4} k_{\mathrm{SGS}}\left(b_{i k} \widetilde{S}_{j k}+b_{j k} \tilde{S}_{i k}-\frac{2}{3} b_{l m} \widetilde{S}_{l m} \delta_{i j}\right) \\
& +g_{5} k_{\mathrm{SGS}}\left(b_{i k} \widetilde{\Omega}_{j k}+b_{j k} \widetilde{\Omega}_{i k}\right)
\end{aligned}
$$

where $\widetilde{S}_{i j}=1 / 2\left(\partial_{j} \widetilde{U}_{i}+\partial_{i} \widetilde{U}_{j}\right)$ and $\widetilde{\Omega}_{i j}=1 / 2\left(\partial_{j} \widetilde{U}_{i}-\partial_{i} \widetilde{U}_{j}\right)$ are, respectively, the rate of strain and rotation tensor based on the resolved velocity, and $b_{i j}=\tau_{i j} /\left(2 k_{\mathrm{SGS}}\right)-\delta_{i j} / 3$ is the SGS anisotropy tensor. The constants are $g_{1}=3.4, g_{1}^{*}=1.8, g_{3}=0.8$, $g_{3}^{*}=1.3, g_{4}=1.25$, and $g_{5}=0.4$. Obviously, the ensembleaveraged quantities used in RANS are replaced by the filtered quantities. Considering that a RANS model for the pressure term can be used without modification in the filtered equations is a strong assumption, but in the absence of DNS data providing the budgets of the subgrid-stress transport equation, this is a reasonable choice. A recalibration of the coefficient would probably improve the accuracy of the model, but such a procedure also requires an appropriate DNS database. Chaouat and Schiestel ${ }^{14}$ proposed a modification of the coefficient of the slow part of the pressure term function of the cutoff wavenumber, in order to increase the return to isotropy in the inertial range. The influence of such a modification in the present model [first term in the right hand side of Eq. (42)] is investigated in Sec. IV D.

\section{Dissipation rate}

In hybrid RANS/LES, the cutoff wavenumber of the filter is supposed to be located far before the dissipative scales, such that the dissipation rate $\varepsilon_{i j_{\mathrm{SGS}}}$ of the subgrid stress $\tau_{i j}$ is, in average, very close to the usual dissipation rate $\varepsilon_{i j}$ of the Reynolds stress $\overline{u_{i} u_{j}}$, i.e., $\overline{\varepsilon_{i j} j_{\mathrm{SGS}}} \simeq \varepsilon_{i j}$. The assumption of isotropy of the dissipation is a matter of debate, ${ }^{53}$ but in RANS second-moment closures, $\varepsilon_{i j}$ is usually modeled by the isotropic expression $\frac{2}{3} \varepsilon \delta_{i j}$, and the departure from isotropy (the deviatoric part) is assumed to be contained in the model for the slow part of the redistribution term. ${ }^{54}$ Although $\varepsilon_{i j_{\mathrm{SGS}}}$, the dissipation rate of the subgrid stress, is likely to be more anisotropic than its average $\varepsilon_{i j}$, in the absence of a DNS database for the budgets of the subgrid stress, the abovementioned decomposition between the deviatoric part, grouped together with the slow part of the pressure term $\phi_{i j_{\mathrm{SGS}}}$, and the isotropic part $\frac{2}{3} \varepsilon_{\mathrm{SGS}} \delta_{i j}$, is considered as valid, at least far away from the wall. Moreover, a recalibration of the slow part is considered in Sec. IV D.

Near the wall, the hybrid model is in RANS mode, and thus a RANS model can be used. In order to extend the validity of the SSG model ${ }^{29}$ to the near-wall region, Manceau and Hanjalić ${ }^{27}$ showed that the model of Rotta, ${ }^{55}$ $\varepsilon_{i j}=\varepsilon \overline{u_{i} u_{j}} / k$, can be associated with the near-wall form [Eq. (41)] of the pressure term, such that the exact asymptotic behavior of the Reynolds stress is satisfied. Moreover, following the RANS practice, ${ }^{27}$ in order to bridge the near-wall and far away from the wall forms of the dissipation rate tensor, similarly to the pressure term, the two forms of the model are combined, leading to 


$$
\varepsilon_{i j_{\mathrm{SGS}}}=(1-f) \frac{\tau_{i j}}{k_{\mathrm{SGS}}} \varepsilon_{\mathrm{SGS}}+f \frac{2}{3} \varepsilon_{\mathrm{SGS}} \delta_{i j} .
$$

The dissipation rate involved in Eq. (43) is provided by the transport equation

$$
\begin{aligned}
\frac{\tilde{D} \varepsilon_{\mathrm{SGS}}}{\widetilde{D} t}= & C_{\varepsilon_{1}}^{\prime} \frac{P_{\mathrm{SGS}}}{T}-C_{\varepsilon_{2}}^{*} \frac{\varepsilon_{\mathrm{SGS}}}{T} \\
& +\frac{\partial}{\partial x_{l}}\left(\nu \delta_{l m}+C_{e} T \tau_{l m}\right) \frac{\partial \varepsilon_{\mathrm{SGS}}}{\partial x_{m}} .
\end{aligned}
$$

Following the PITM methodology described in Sec. III A, the $C_{\varepsilon_{2}}^{*}$ coefficient is dependent on the cutoff wavenumber [Eq. (18)], with $C_{\varepsilon_{1}}=1.44$ and $C_{\varepsilon_{2}}=1.83$. Since the flow considered will be inhomogeneous, transport terms have been incorporated. The turbulent diffusion term is modeled by the generalized gradient diffusion hypothesis, ${ }^{56}$ with $C_{e}=0.18$. Following Manceau and Hanjalić, ${ }^{27}$ to avoid singularities at the wall, the subgrid time scale is bounded by the Kolmogorov scale

$$
T=\max \left(\frac{k_{\mathrm{SGS}}}{\varepsilon_{\mathrm{SGS}}}, C_{T} \sqrt{\frac{\nu}{\varepsilon_{\mathrm{SGS}}}}\right),
$$

with $C_{T}=6.0$, and to take into account the increase in the production of dissipation in the near-wall zone, the coefficient of the generation term in the dissipation equation is taken as

$$
C_{\varepsilon_{1}}^{\prime}=C_{\varepsilon_{1}}\left(1+A_{1}(1-f) \sqrt{\frac{k_{\mathrm{SGS}}}{\tau_{i j} n_{i} n_{j}}}\right),
$$

with $A_{1}=0.03$ and $n$ being the generalized wall-normal vector. This formulation gives the classical value $C_{\varepsilon_{1}}$ far from the wall, and a larger value in the near-wall zone. The boundary condition at the wall (subscript $w$ ) for the dissipation rate is

$$
\varepsilon_{\mathrm{SGS} w}=\lim _{x_{n} \rightarrow 0} 2 \nu \frac{k_{\mathrm{SGS}}}{x_{n}^{2}}
$$

where $x_{n}$ is distance to the wall.

\section{Turbulent diffusion}

The generalized gradient diffusion hypothesis of Daly and Harlow ${ }^{56}$ is extended to the diffusion process of the SGS stresses

$$
D_{i j_{\mathrm{SGS}}}^{T}=\frac{\partial}{\partial x_{l}}\left(C_{d} T \tau_{l m} \frac{\partial \tau_{i j}}{\partial x_{m}}\right),
$$

where $C_{d}=0.21$ and the time scale is given by Eq. (45).

\section{Modeling issues}

As mentioned above, some modeling issues remain and must be discussed. Some of them are related to the PITM methodology and others are due to the development of an elliptic-blending model in the hybrid context.

- The value of the RANS-LES transition parameter $r$ must be chosen as a function of the cutoff wavenum- ber in such a way that it is consistent with both RANS and LES. The issue of explicitly relating $r$ to the cutoff wavenumber is addressed in Sec. IV B.

- The elliptic relaxation equation (39) enables to account for the nonviscous, nonlocal blocking effect of the wall on the subgrid stress. This kinematic effect reflects the incompressibility condition for the nonresolved scales. In a hybrid context, the blocking of the large scales, which are explicitly resolved, follows from the explicit resolution of the continuity equation $\left(\partial_{k} \widetilde{U}_{k}=0\right)$. The elliptic blending aims at imposing the blocking effect only on the modeled scales, which implies that the correlation length scale $L_{\mathrm{SGS}}$, entering Eq. (39), must be decreased compared to the RANS case, where all the scales of motion are modeled. This issue is addressed in Sec. IV C.

- It is usual to assume that small scales return to isotropy faster than the large scales (e.g., Hinze ${ }^{57}$ or Pope $^{58}$ ), which could be reproduced, as suggested by Chaouat and Schiestel, ${ }^{14}$ by making the slow part of the pressure term a function of the filter width. The necessity of doing such a modification is addressed in Sec. IV D.

To investigate these issues, the test case of a channel flow is considered at $\operatorname{Re}_{\tau}=u_{\tau} h / \nu=395$, where $h$ is the channel half-width and $u_{\tau}$ the friction velocity, and results are compared to DNS data. ${ }^{38}$ The streamwise, wall-normal, and spanwise directions are, respectively, denoted by $\mathbf{x}, \mathbf{y}$, and $\mathbf{z}$. In all the following figures, the quantities will be presented in wall units, the reference velocity and length scale being $u_{\tau}$ and $\nu / u_{\tau}$, respectively.

\section{INVESTIGATION OF THE MODELLING ISSUES}

\section{A. Numerical method}

Computations are performed with Code_Saturne, a parallel, finite volume solver on unstructured grids, developed at EDF. ${ }^{59}$ Spatial discretization is based on a collocation of all the variables at the center of gravity of the cells. Velocity/pressure coupling is ensured by the SIMPLEC algorithm, with a Rhie and Chow interpolation in the pressurecorrection step (e.g., Ferziger and Peric ${ }^{60}$ ). The Poisson equation is solved with a conjugate gradient method. Time advancement is based on a Crank-Nicolson scheme. Spatial derivatives are approximated by a second-order centraldifference scheme for the resolved velocity field and a firstorder upwind-difference scheme for the subgrid turbulence field.

The computational domain is $(8 h, 2 h$, and $4 h)$ long in streamwise, wall-normal, and spanwise directions, respectively, corresponding to $(3160,790$, and 1580$)$ in wall units. The reference mesh $\mathrm{M}^{0}$ contains $64 \times 42 \times 64=172032$ cells. This mesh is chosen too coarse $\left(\Delta x^{+}=50, \Delta z^{+}=25\right)$ in the near-wall region to perform a well-resolved LES, which requires with the present second-order numerical method $\Delta x^{+}=20$ and $\Delta z^{+}=10$, but sufficiently fine and isotropic in the outer region to enable the model to approach the LES mode. One of the important features expected from a hybrid 
TABLE I. Value of $\beta_{0}$ as a function of the parameter $C_{g}$ [see Eq. (51)].

\begin{tabular}{lllllc}
\hline \hline$C_{g}$ & 2 & 4 & 6 & 8 & 10 \\
$\beta_{0}$ & 0.44 & 0.28 & 0.21 & 0.18 & 0.15 \\
\hline \hline
\end{tabular}

RANS-LES model is the ability to safely and progressively return to the RANS solution when the mesh is coarsened. In order to assess the behavior of the model in such a case, a second mesh, $\mathrm{M}^{-}$, is designed, by reducing the number of cells in the streamwise and spanwise directions by a factor of 2 .

Due to the rapid variations in space and time of the resolved field, it is found necessary to average the strain tensor in the homogeneous directions, before evaluating the source terms of the subgrid-stress transport equations, similar to what was done by Chaouat and Schiestel,,${ }^{14}$ to sustain fluctuations in the resolved field. This procedure is used only to aid the development of the model, in order to test the different modeling issues. Since it is not physically justified, the last section of this article ( $\mathrm{Sec} . \mathrm{V})$, is devoted to the development of a dynamic procedure, aiming at helping the model to sustain the expected amount of resolved energy. Although the theory provides the link [Eq. (18)] between the coefficient $C_{\varepsilon_{2}}^{*}$ and the ratio modeled energy over total energy $(r)$, the ratio observed in the simulations is not necessarily the expected ratio.

\section{B. Modeling of the parameter $r$}

The first modeling issue concerns the choice of the parameter $r$, which is the ratio of the modeled to the total energy. This ratio enters the evaluation of the $C_{\varepsilon_{2}}^{*}$ coefficient in the dissipation equation [Eqs. (44) and (18)]. The filter width is related to the local mesh size $\Delta=(\Delta x \Delta y \Delta z)^{1 / 3}$ by $\Delta_{f}=C_{g} \Delta / 2$, where $C_{g} \geq 2$ is a constant, such that the cutoff wavenumber can be written as

$$
\kappa_{c}=\frac{2 \pi}{C_{g} \Delta}
$$

Using definition (49), and introducing for convenience the dimensionless cutoff wavenumber $\eta_{c}$

$$
\eta_{c}=\frac{\pi}{\Delta} \frac{k^{3 / 2}}{\varepsilon}
$$

Eq. (19) can be written as

$$
r=\frac{1}{\beta_{0} \eta_{c}^{2 / 3}} \quad \text { where } \quad \beta_{0}=\frac{2}{3 C_{K}}\left(\frac{2}{C_{g}}\right)^{2 / 3} \leq 0.44 .
$$

Table I gives the value of $\beta_{0}$ as a function of the parameter $C_{g}$. The relevant value of $C_{g}$ will be investigated at the end of this section. Equation (51) is not compatible with the RANS limit $\left(\lim _{\eta_{c} \rightarrow 0} r=1\right)$, simply because the Kolmogorov $-5 / 3$ power law is not valid at large scales. A first possibility to correct this shortcoming is to bound $r$,

$$
r_{\mathrm{MIN}}=\min \left(1 ; \frac{1}{\beta_{0} \eta_{c}^{2 / 3}}\right) .
$$

This formulation will be denoted as MIN hereafter. Some authors ${ }^{13,14}$ proposed another simple empirical choice

$$
r_{\mathrm{CS}}=\frac{1}{1+\beta_{0} \eta_{c}^{2 / 3}} \text {. }
$$

Formulation (53) will be denoted as CS hereafter. Schiestel and Dejoan ${ }^{14}$ proposed the use of the von Karman spectrum to evaluate $r$

$$
E(\kappa)=C_{K} \varepsilon^{2 / 3} \kappa^{q}\left[\frac{C_{K}}{q+1}\left(\frac{\varepsilon}{k^{3 / 2}}\right)^{m-1}+\kappa^{m-1}\right]^{-(m+q) /(m-1)},
$$

with $m=5 / 3$. The total fluctuating kinetic energy $k$ is given by the integration of Eq. (54) between $\kappa=0$ and $\kappa=\infty$. Note that in decaying turbulence, the dissipation rate is given by the time derivative of the kinetic energy, i.e., $\varepsilon=-d k / d t$, and that $d \varepsilon / d t=-C_{\varepsilon_{2}} \varepsilon^{2} / k$. Combining these results, the following relation can be obtained (see Schiestel and Dejoan ${ }^{13}$ for more details):

$$
C_{\varepsilon_{2}}=\frac{3 q+5}{2(q+1)},
$$

which gives $q \simeq 2.03$ for $C_{\varepsilon_{2}}=1.83$. The parameter $r$ is evaluated by integration of Eq. (54) between $\kappa=\kappa_{c}$ and $\kappa=\infty$, and is denoted as VK

$$
r_{\mathrm{VK}}=1-\left[1+\frac{2}{3(q+1) \beta_{0}} \eta_{c}^{-2 / 3}\right]^{-(3 / 2)(q+1)} .
$$

It can be easily seen that this formulation is compatible with the RANS limit. Table II gives a summary of the different formulations for the parameter $r$ tested in the present work.

The integral length scale $k^{3 / 2} / \varepsilon$ enters the evaluation of the parameter $r$ through the dimensionless wavenumber $\eta_{c}$.

TABLE II. Different semiempirical proposals for the parameter $r$.

\begin{tabular}{lc}
\hline \hline Acronym & Formulation \\
\hline MIN & $r=\min \left(1 ; \frac{1}{\beta_{0}} \eta_{c}^{-2 / 3}\right)$ \\
VK & $r=1-\left[1+\frac{2}{3(q+1) \beta_{0}} \eta_{c}^{-2 / 3}\right]^{-(3 / 2)(q+1)}$ \\
CS & $r=\frac{1}{1+\beta_{0} \eta_{c}^{2 / 3}}$ \\
EB & $r=\min \left[1 ;\left(1-\alpha^{2}\right)+\alpha^{2} \frac{1}{\beta_{0}} \eta_{c}^{-2 / 3}\right]$
\end{tabular}




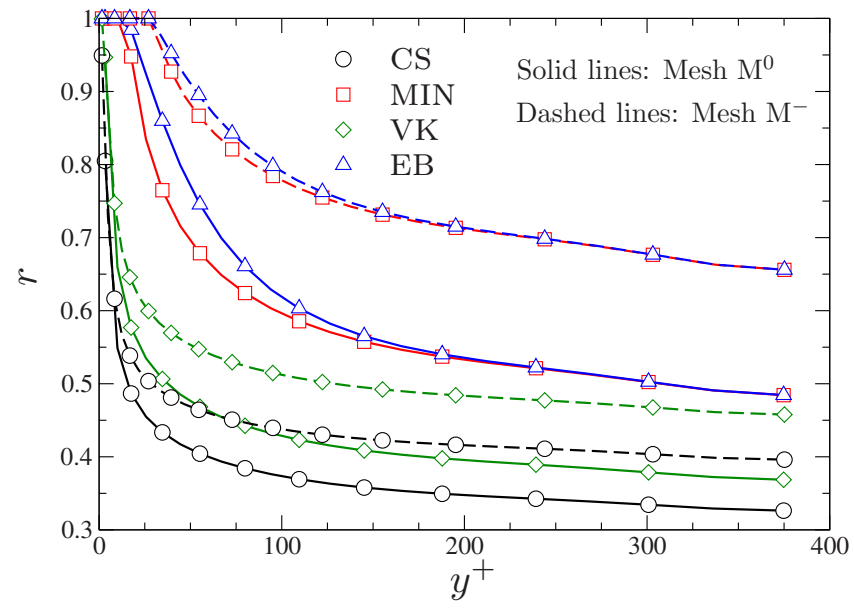

FIG. 2. (Color online) Profile of the parameter $r$ given by the different formulations for $\beta_{0}=0.20$. See Table II for the definition of the acronyms.

However, the total turbulent kinetic energy $k$ is not known at the beginning of the computation. In the present section, the integral scale is evaluated from a preliminary RANS computation performed with the EB-RSM. This approach is not problematic in a channel flow because the length scales given by the average PITM solution are close to those given by the RANS model. However, in other kind of flows, such as massively separated flows, the averaged PITM results are expected to be different from the RANS results. In such flows, it will be crucial, after a transient phase, to update the integral length scale during the computation. Therefore, the evaluation of the length scale during the computation will be introduced once the final form of the model is chosen, i.e., in Sec. V.

Figure 2 shows the profile of $r$ for the different formulations. Using formulation CS for $r$ with mesh $\mathrm{M}^{-}$, it is noticed that the condition $\beta_{0} \eta_{c}^{2 / 3} \gg 1$ is not satisfied at the center of the channel, such that the formulation (51), based on the Kolmogorov law, is not asymptotically approached. Figure 3 shows the influence of the particular form of $r$ on the prediction of the resolved, modeled, and total streamwise component of the Reynolds stress for the mesh $\mathrm{M}^{-}$. It is observed that the resolved part of the Reynolds stress increases very rapidly as a function of the distance to the wall and is strongly overestimated, even on this coarse mesh. The same results are obtained with formulation VK (not shown here). This behavior seems to be related to the fact that formulations CS and VK do not involve the distance to the wall, and thus the transition RANS-LES completely relies on the mesh, and can occur too close to the wall. The same problem is faced with the original DES formulation, which motivated the development of delayed detached-eddy simulation (DDES), ${ }^{61}$ in order to avoid artifacts such as mesh-induced separation.

In the elliptic-blending framework, it is proposed to blend the value of $r$ near the wall $(r=1)$ and its theoretical value, given by Eq. (51), valid far from the wall, as

$$
r_{\mathrm{EB}}=\min \left[1 ;\left(1-\alpha^{p}\right)+\alpha^{p} \frac{1}{\beta_{0} \eta_{c}^{2 / 3}}\right],
$$

where $\alpha$ is the blending parameter solution of Eq. (39) and $p$ is a positive constant. Formulation (57), denoted EB hereafter, enables a better control of the transition RANS-LES, as shown on Figs. 2 and 3 because $r$ is not only a function of the local cell size, but also of the distance to the wall, through the parameter $\alpha$. Using the exact asymptotic behavior at the wall of the different quantities in a fully developed channel flow, i.e., $k \sim y^{2}, \varepsilon \sim 1$, and $\alpha \sim y$, and assuming that $\Delta \sim y^{n}$, it is easy to show that $\eta_{c} \sim y^{3-n}$ and thus

$$
\alpha^{p} \frac{1}{\beta_{0} \eta_{c}^{2 / 3}} \sim y^{s},
$$

where $s=p-\frac{2}{3}(3-n)$. In order to have a correct asymptotic behavior of $r$ at the wall in Eq. (57) (i.e., $r \rightarrow 1$ ), $s>0$ must be imposed, which leads to

$$
p>\frac{2}{3}(3-n) \text {. }
$$

Since the near-wall zone is to be solved in RANS mode, it can be assumed that $\Delta x \sim 1, \Delta z \sim 1$, and $\Delta y \sim y$ (near-wall clustering), implying $n=1 / 3$ and $p>16 / 9$. For simplicity, $p$ is chosen as an integer $p=2$. For this value of $p$, it is noticed that Eq. (59) is satisfied for any positive value of $n$.

With formulations VK and CS, $r$ decreases very quickly with the wall distance, and thus, the transition to a LES calculation is too close to the wall, explaining the severe overestimation of the Reynolds stress (see Fig. 3). Formulations MIN and EB are very similar in the case considered here, and have a satisfactory behavior in the vicinity of the wall. Formulation EB will be chosen for all the tests done in the following sections.

A range of values have been tested for the $C_{g}$ parameter, which enters the evaluation of $\beta_{0}$ [Eq. (51)]. Indeed, the highest wavenumber that can be obtained on a given mesh depends on the numerical scheme, and Ghosal ${ }^{62}$ recommends the value $C_{g}=6$ for a second-order central-difference scheme. For large values of $C_{g}\left(C_{g}>10\right), r=1$ is obtained all across the channel (with the formulation EB for $r$ ), leading to a RANS solution. When $C_{g}$ is too low, the cutoff wavenumber is increased, leading to a decrease in the modeled part of the Reynolds stress and a consequent increase in the resolved part, especially near the walls. Thus, for $C_{g}<4.5$, the total Reynolds stresses are strongly overestimated. In the range $[4.5,10]$, the turbulence statistics are weakly dependent on the value of $C_{g}$. The optimal value is found to be $C_{g} \simeq 6.5$, corresponding to $\beta_{0}=0.20$, which is very close to the value suggested by Ghosal. ${ }^{62}$

\section{Length scale for the wall-blocking effect}

The second modeling issue concerns the choice of the correlation length scale $L_{\text {SGS }}$ for the wall effects. Theoretical (e.g., Hunt and Graham ${ }^{63}$ ) and DNS studies ${ }^{64}$ showed that the structures of the flow, and the associated length scales, are strongly affected by the presence of a solid boundary even in the absence of mean shear because of the blocking 

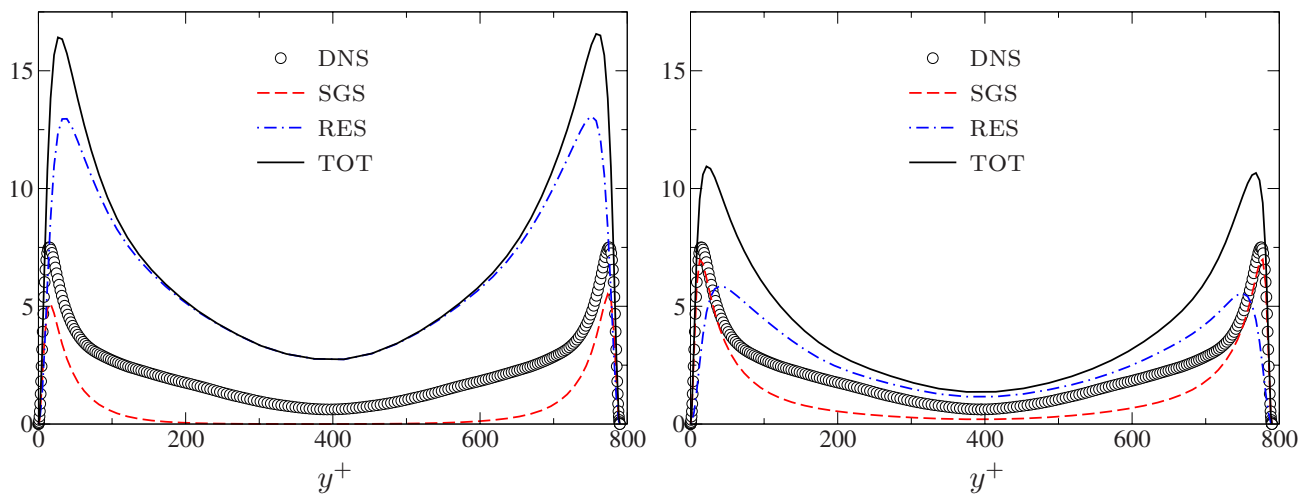

FIG. 3. (Color online) Influence of the form of $r$ on the resolved $\left(\mathrm{RES}=\overline{u^{\prime} u^{\prime}}\right.$ ), modeled $\left(\mathrm{SGS}={\overline{\tau_{11}}}^{+}\right)$, and total $\left(\mathrm{TOT}=R_{11}^{+}=\mathrm{RES}+\mathrm{SGS}\right)$ streamwise component of the Reynolds stress. Mesh $\mathrm{M}^{-}, \beta_{0}=0.20$. Left: formulation CS [Eq. (53)]. Right: formulation EB [Eq. (57)].

effect, which is nonlocal. Indeed, through the pressure field, the kinematic impermeability condition at the wall affects the flow up to an integral scale from the wall. ${ }^{63}$ The length scale $L_{\mathrm{SGS}}$ in Eq. (39) characterizes the distance at which the nonlocal kinematic blocking of the wall is felt by the nonresolved motion. On the contrary, this effect is explicitly imposed on the resolved scales via the resolution of the continuity equation. Therefore, in the hybrid context, the length scale of the nonresolved fluctuations is dependent on the filter width, and the modeling of the length scale must be consequently modified. $L_{\mathrm{SGS}}$ must be smaller than its RANS counterpart $L$, in order to characterize only the modeled scales.

In a RANS framework, the elliptic relaxation equation [Eq. (39)] is solved, with the correlation length scale given by

$$
L=C_{L} \max \left(\frac{k^{3 / 2}}{\varepsilon}, L_{b}\right) .
$$

$L_{b}$ is related to the Kolmogorov scale $L_{\eta}$ by $L_{b}=C_{\eta} L_{\eta}$. The coefficients are $C_{L}=0.161$ and $C_{\eta}=80$. In order to illustrate the influence of this length scale, it is noted that the solution of Eq. (39) can be well approximated by

$$
\alpha(y)=1-\exp \left(-\frac{y}{L_{\mathrm{SGS}}}\right),
$$

with a constant length scale, where $L_{\text {SGS }}$ must be lower than the admissible value for a RANS computation. Figure 4 shows the profile of the blending coefficient $\alpha$ calculated with the EB-RSM in a RANS framework. It can be seen that using the constant length scale $L=0.06 h$ yields a blending coefficient very close to the one obtained using Eq. (60). When the length scale is reduced to $L=0.04 h, \alpha$ reaches the asymptotic value $\alpha=1$ closer to the wall, such that the wall blockage, active where $\alpha<1$, is reduced. The effect of this reduction on the anisotropy is shown in Fig. 5 by comparing the wall-normal component of the Reynolds stress (resolved, modeled, and total), obtained with $L_{\mathrm{SGS}}=0.04 h$ and $0.06 h$. It is seen that the decrease in $L_{\mathrm{SGS}}$ modifies the anisotropy, by reducing the blocking effect, i.e., the inhibition of the redistribution from $\tau_{11}$ to $\tau_{22}$. As expected, the blocking effect only affects the SGSs, leaving the resolved scales almost unchanged. Reducing further the correlation length scale is attractive, since $R_{22}$ is underestimated. However, Fig. 6, which compares the velocity profiles obtained with three values of $L_{\mathrm{SGS}}$, illustrates the fact that this length scale also influences the mean velocity profile, through the blocking imposed on $\tau_{12}$.

By artificially imposing a constant length scale $L_{\mathrm{SGS}}$, it has been shown that in the hybrid formulation, it is necessary to reduce this length compared to the RANS value. This reduction can be achieved in a natural way, by replacing the integral length scale $k^{3 / 2} / \varepsilon$ in Eq. (60) by the length scale characterizing the largest subgrid eddies $k_{\mathrm{SGS}}^{3 / 2} / \varepsilon_{\mathrm{SGS}}$. When the cutoff wavenumber increases, the scale of the largest subgrid eddies decreases, such that the region over which the blocking effect of the wall is felt by the SGSs progressively vanishes, according to Eq. (39). This behavior is consistent with the analysis of Sec. III B 1 a, in particular Eq. (35), in which $L_{\mathrm{SGS}}$ is defined from the two-point correlations for the SGSs. As the integral length scale $k^{3 / 2} / \varepsilon$ is decreased by a factor $r^{3 / 2}$ to obtain $k_{\mathrm{SGS}}^{3 / 2} / \varepsilon_{\mathrm{SGS}}$ in the hybrid context, it is proposed to decrease the scale $L_{b}$ by the same factor, leading to

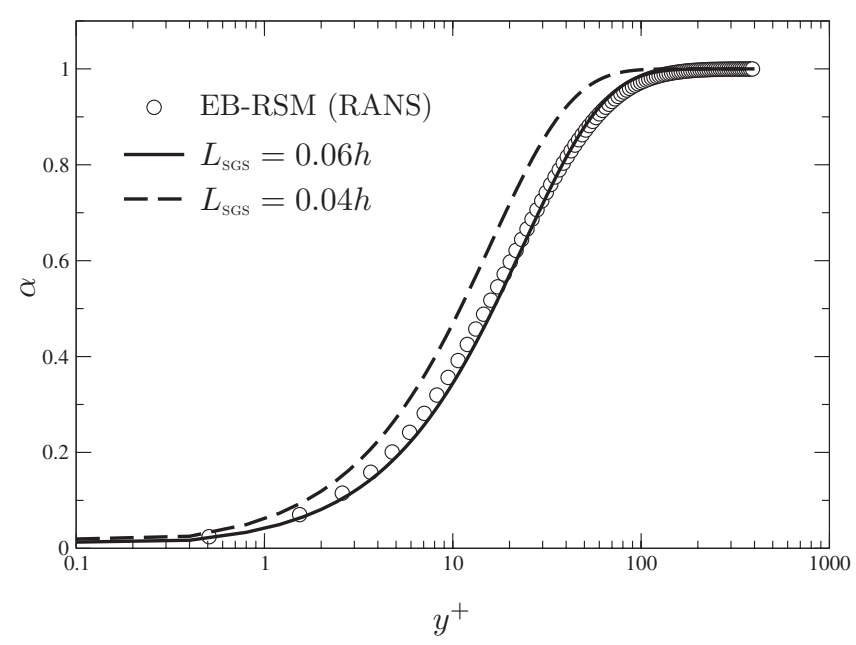

FIG. 4. Profile of the blending coefficient $\alpha$ calculated with the EB-RSM in a RANS framework. Comparison with Eq. (61) with $L_{\mathrm{SGS}}=0.06 h$ and $L_{\mathrm{SGS}}=0.04 h$. 

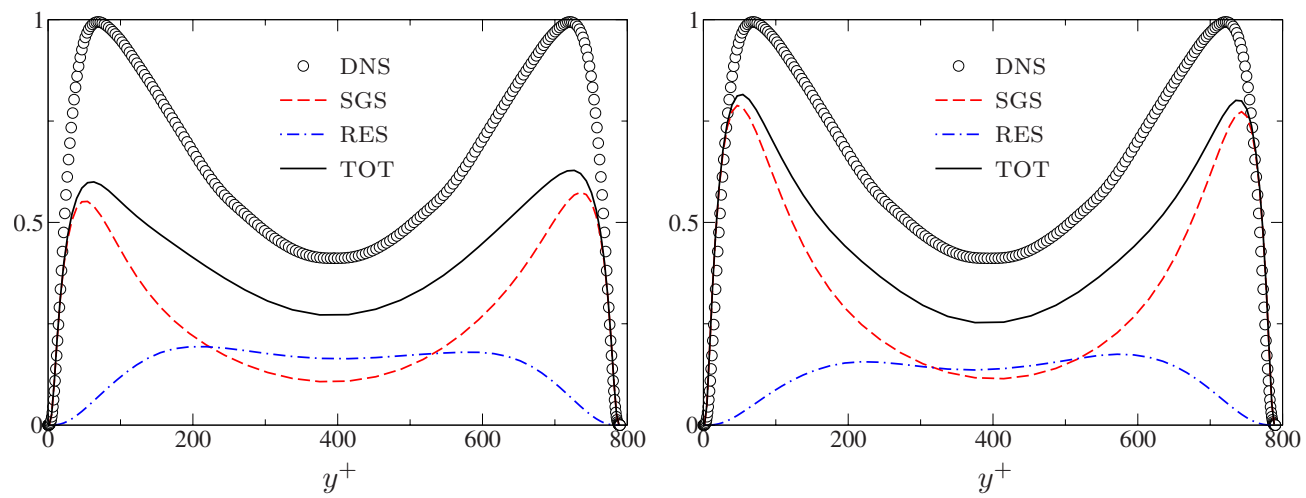

FIG. 5. (Color online) Influence of $L_{\mathrm{SGS}}$ on the resolved $\left(\mathrm{RES}=\overline{v^{\prime} v^{\prime}}\right.$ ), modeled $\left(\mathrm{SGS}={\overline{\tau_{22}}}^{+}\right.$), and total $\left(\mathrm{TOT}=R_{22}^{+}=\mathrm{RES}+\mathrm{SGS}\right)$ wall-normal component of the Reynolds stress. Left: $L_{\mathrm{SGS}}=0.06 h$ (RANS value). Right: $L_{\mathrm{SGS}}=0.04 h$.

$$
L_{\mathrm{SGS}}=C_{L} \max \left(\frac{k_{\mathrm{SGS}}^{3 / 2}}{\varepsilon_{\mathrm{SGS}}}, r^{3 / 2} C_{\eta} \frac{\nu^{3 / 4}}{\varepsilon_{\mathrm{SGS}}^{1 / 4}}\right)
$$

At the RANS limit $(r=1)$, Eq. (60) is recovered.

Using formulation (62), Figs. 7 and 8 show the contribution of the resolved and modeled scales to the total shear stress and turbulent energy, respectively. It can be seen that near the wall, the SGS part is dominant (RANS mode) and decreases toward the center of the channel, where the resolved part in turn becomes dominant (LES mode). When the mesh is coarsened, the cutoff wavenumber, proportional to the inverse of the cell size, is decreased, and the balance modeled/resolved contributions is modified as expected: the resolved large-scale part decreases and the modeled SGS part increases. However, Figs. 7 and 8 also show that the contribution of the SGSs is very small on the reference mesh $\mathrm{M}^{0}$, leading to a pseudo-DNS (coarse DNS) at the center of the channel. This behavior is identified to be due to the artificial averaging in the homogeneous directions of the sources terms of the subgrid-stress transport equations: with the dynamic procedure presented in Sec. V, the model will exhibit a higher level of subgrid energy.

Figure 9 shows the normal components of the Reynolds stress obtained using this formulation [Eq. (62)]. It is observed that the normal stresses are globally well reproduced, even though the streamwise component is overestimated in

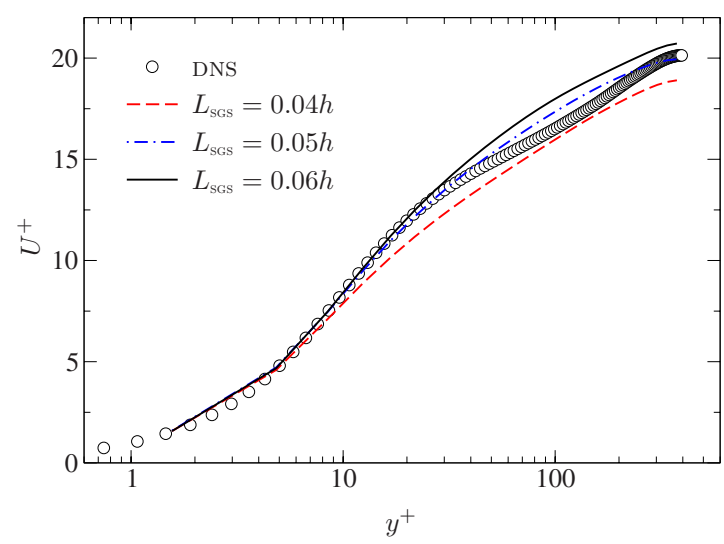

FIG. 6. (Color online) Influence of $L_{\mathrm{SGS}}$ on the mean velocity profile. Mesh $\mathrm{M}^{-}$and $\alpha$ given by Eq. (61). the central part of the channel, and the wall-normal and spanwise component are underestimated. The main objective of the introduction of elliptic blending in the model is reached, i.e., the blockage of the wall-normal fluctuations, which is necessary to obtain a correct reproduction of the anisotropy in the near-wall region.

\section{Modification of the pressure term}

It is usual to assume that the small scales return to isotropy faster than the large scales. When the cutoff wavenumber is in the inertial range of the turbulent energy spectrum, the structures can be considered as isotropic with a good accuracy (e.g., Hinze ${ }^{57}$ or Pope ${ }^{58}$ ). In the model, the pressure term is decomposed into a rapid part, depending directly on the velocity field, and a slow part. It is worth recalling that the model for the slow part also accounts for the deviatoric part of the dissipation term, as mentioned in Sec. III B 2. In the hybrid context, Chaouat and Schiestel ${ }^{14}$ suggested a modification of the slow part in order to increase the return to isotropy of the small scales. Following Schiestel, ${ }^{65}$ they proposed to introduce an empirical parameter $f_{\mathrm{SGS}} \geq 1$ function of the cutoff wavenumber, with $\lim _{\eta_{c} \rightarrow 0} f_{\mathrm{SGS}}=1$, in order to remain consistent with the RANS limit.

It is recalled that in the elliptic-blending framework, the pressure term $\phi_{i j_{\mathrm{SGS}}}$ is decomposed into the homogeneous contribution $\phi_{i j}^{h}$, valid away from the wall, and the near-wall contribution $\phi_{i j}^{w}$, as shown by Eq. (40). The former, given here by the SSG model ${ }^{29}$ [Eq. (42)], can be decomposed into a rapid part $\phi_{i j}^{h, r}$ and a slow part $\phi_{i j}^{h, s}$, given by

$$
\phi_{i j}^{h, s}=-\left(g_{1}+g_{1}^{*} \frac{P_{\mathrm{SGS}}}{\varepsilon_{\mathrm{SGS}}}\right) \varepsilon_{\mathrm{SGS}} b_{i j} .
$$

In the hybrid elliptic-blending context, the homogeneous part of the pressure term is modified as

$$
\phi_{i j}^{h}=f_{\mathrm{SGS}} \phi_{i j}^{h, s}+\phi_{i j}^{h, r} .
$$

Since the aim of the hybrid methodology is to perform a RANS calculation near the wall, $\phi_{i j}^{w}$ does not require any modification compared to the RANS model.

Two formulations of $f_{\mathrm{SGS}}$ have been tested in the present work. The first one, denoted as P-CS, was proposed by Chaouat and Schiestel ${ }^{14}$ 

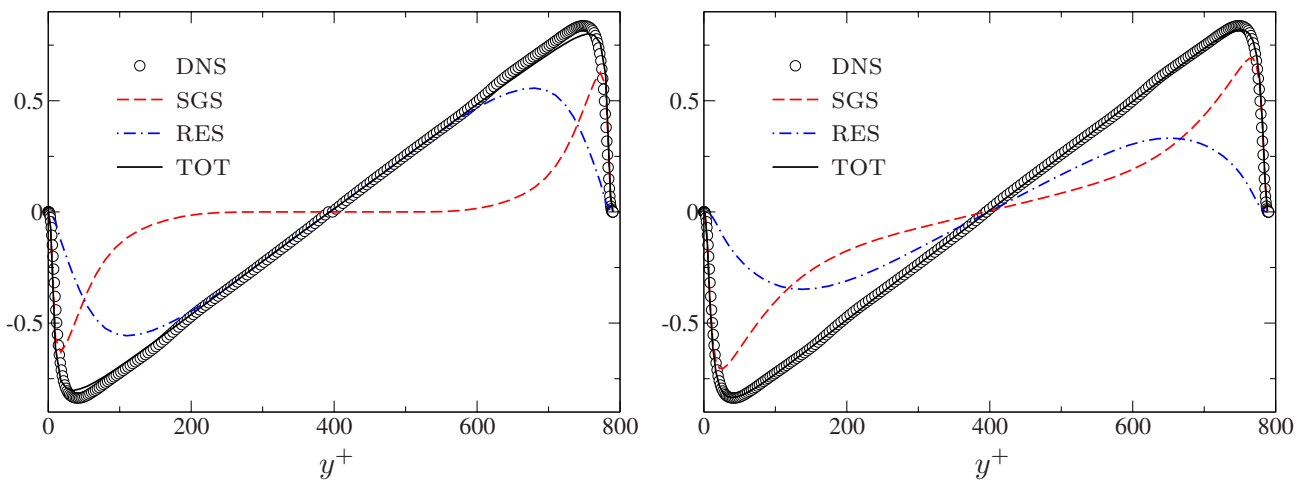

FIG. 7. (Color online) Profile of resolved $\left(\mathrm{RES}=\overline{u^{\prime} v^{\prime}}\right.$ ), modeled $\left(\mathrm{SGS}={\overline{\tau_{12}}}^{+}\right.$), and total (TOT $\left.=R_{12}^{+}=\mathrm{RES}+\mathrm{SGS}\right)$ shear stress. Formulation EB for $r$ and $L_{\mathrm{SGS}}$ given by Eq. (62). Left: mesh $\mathrm{M}^{0}$; Right: mesh $\mathrm{M}^{-}$.

$$
f_{\mathrm{SGS}}=\frac{1+\gamma \eta_{c}^{2}}{1+\eta_{c}^{2}}
$$

where $\gamma=1.5$ is a constant originating from the work of Schiestel. ${ }^{65,66}$ Compatibility is guaranteed with the RANS limit. In the LES regions, where $\eta_{c}$ is large, $f_{\mathrm{SGS}}$ tends to $\gamma$, in order to increase the return to isotropy of the SGSs. The profile of $f_{\mathrm{SGS}}$ is given in Fig. 10. It is noticed that $f_{\mathrm{SGS}}$ reaches its maximum value very quickly and too close to the wall, and that the total Reynolds stress is overestimated, due to high values of the resolved part near the wall (figure not shown here). To better control the variations in $f_{\mathrm{SGS}}$ in the vicinity of the wall, similar to Eq. (57), the following form is proposed:

$$
f_{\mathrm{SGS}}=\max \left[1 ;\left(1-\alpha^{b}\right)+\alpha^{b} \frac{\gamma \eta_{c}^{2}}{1+\eta_{c}^{2}}\right]
$$

where $b>0$ is a constant. This formulation is denoted as P-EB. The different proposals for $f_{\mathrm{SGS}}$ tested in the present work are summarized in Table III. In order to have a real effect on the anisotropy, it is found that the value of $b$ must be less than one, and $b=0.5$ is chosen. At the wall, the RANS limit is recovered since $\alpha=0$. Far away from the wall $(\alpha$ $=1), f_{\mathrm{SGS}}$ tends to $\gamma$. Figure 10 compares the profiles of $f_{\mathrm{SGS}}$ obtained with the P-CS formulation and the P-EB formulation with two values of $b$. In Fig. 11, the normal components of the Reynolds stress obtained using the formulation P-EB with $b=0.5$ are shown. Comparing this figure with Fig. 9, it is observed that the normal components of the Reynolds stress are slightly improved at the center of the channel, but at the price of a deterioration of the prediction of the peak value of $R_{11}$ close to the wall. Figure 12 shows the shear stress (resolved, modeled, and total) obtained with the formulation P-EB, for the two meshes. Comparing this figure with Fig. 7, it is seen that, surprisingly, the main effect of the introduction of $f_{\mathrm{SGS}}$ is a significant modification of the balance between resolved and modeled shear stress, in particular at the center of the channel, leading to an extension of the LES region. Actually, by increasing the return to isotropy, the parameter $f_{\mathrm{SGS}}$ tends to decrease the amplitude of the modeled shear stress $-\tau_{12}$. As a consequence, the production of subgrid energy is also decreased. Since the effect on the anisotropy of the normal stresses is marginal, and even detrimental, the use of this modification of the return to isotropy is not recommended in the elliptic-blending framework.

\section{DYNAMIC APPROACH}

The main problem faced by the PITM approach is the difficulty to reach the ratio modeled energy over total energy that the model is supposed to reach: the parameter $r$ entering Eq. (18) provides the model with the energy ratio the user targets, but this value is in general not observed in the solution. In particular, for flows that do not present inflexion
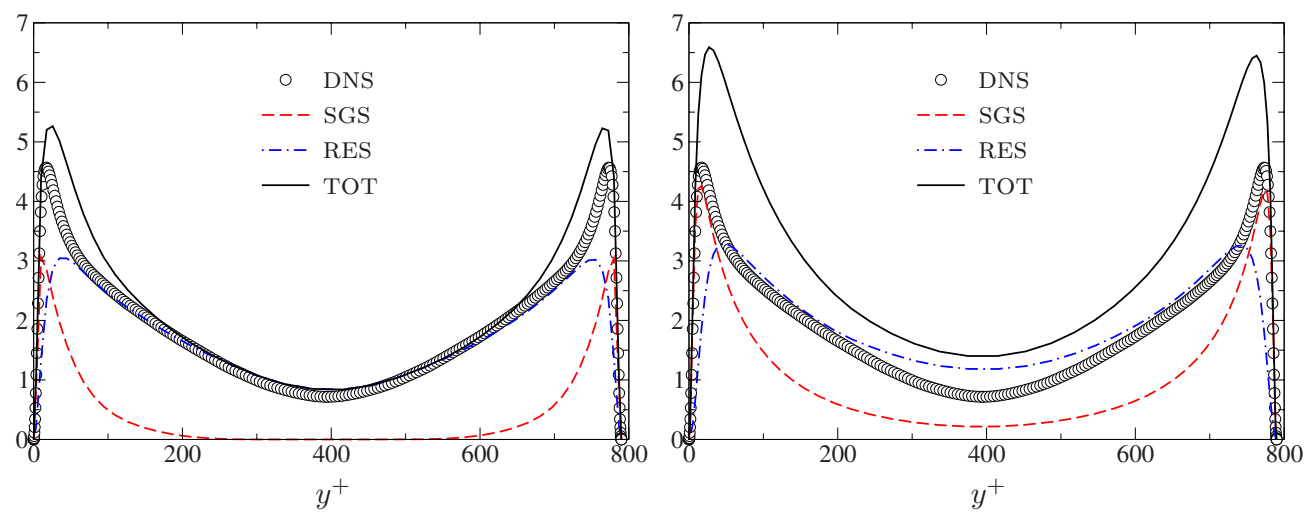

FIG. 8. (Color online) Same figure as Fig. 7 for the turbulent kinetic energy. 


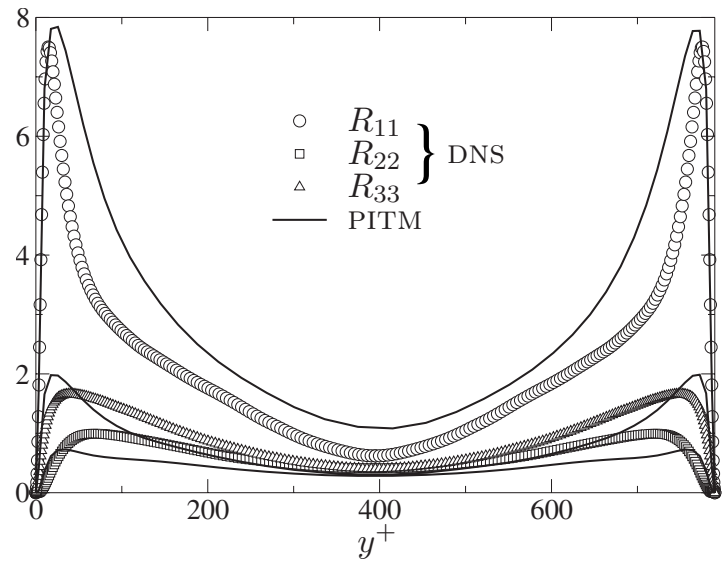

FIG. 9. Profile of the normal components of the Reynolds stress $\left(R_{i j}^{+}\right.$ $=\overline{u_{i}^{\prime} u_{j}^{\prime}}+{\overline{\tau_{i j}}}^{+}$). Formulation EB for $r$ and $L_{\mathrm{SGS}}$ given by Eq. (62). Mesh $\mathrm{M}^{0}$.

points to trigger the growth of fluctuations in the resolved field, such as the present channel flow, the model has a tendency to underestimate the resolved energy, and to eventually tend to a steady solution. This problem is particularly acute at the beginning of the computation, during the transient phase, and sustaining resolved fluctuations during this phase is difficult.

The reason for this behavior probably lies in Eq. (18), which is true in a spatially average sense, since it is derived in spectral space, but not locally. The consequence can be an inappropriate level of dissipation in the SGS energy equation, possibly leading to a rapid decay of the resolved fluctuations. This problem was circumvented in Sec. IV by averaging the sources terms of the subgrid-stress transport equations in homogeneous directions. However, this procedure has been used to aid the development of the new model, but is not justified from a physical point of view, and will not be necessary anymore with the dynamic procedure described below.

Therefore, the present section is devoted to the implementation of a dynamic procedure, whose purpose is twofold: avoiding the decay of the resolved fluctuations during the transient phase of the computation and forcing the model

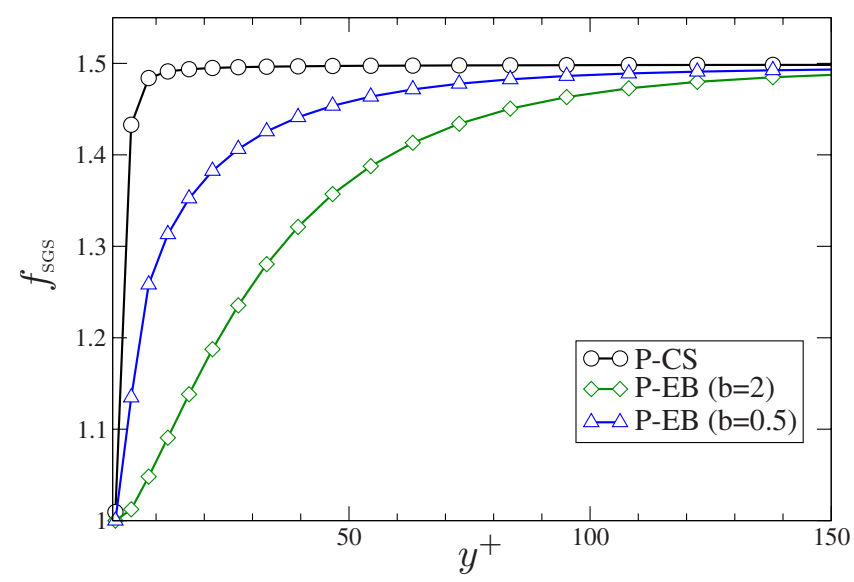

FIG. 10. (Color online) Profile of the function $f_{\mathrm{SGS}}$. See Table III for the meaning of the acronyms.
TABLE III. Different empirical proposals for the function $f_{\mathrm{SGS}}$.

\begin{tabular}{lc}
\hline \hline Acronym & Formulation \\
\hline P-CS & $f_{\mathrm{SGS}}=\frac{1+\gamma \eta_{c}^{2}}{1+\eta_{c}^{2}}$ \\
P-EB & $f_{\mathrm{SGS}}=\max \left[1 ;\left(1-\alpha^{b}\right)+\alpha^{b} \frac{\gamma \eta_{c}^{2}}{1+\eta_{c}^{2}}\right]$ \\
\hline
\end{tabular}

to better approach the expected energy ratio in the permanent state. The latter point is desirable in general, and ensures the internal consistency of the model. Moreover, as was shown in Sec. III A 2, the modification of the length scale entering a RANS model is sufficient to ensure that the turbulent viscosity tends to a SGS viscosity, but this is not true anymore if the observed energy ratio is different from the target energy ratio, since Eq. (21) is not satisfied.

The method simply consists in a dynamical correction of the coefficient $C_{\varepsilon_{2}}^{*}$,

$$
C_{\varepsilon_{2}}^{*}=C_{\varepsilon_{1}}+r\left(C_{\varepsilon_{2}}-C_{\varepsilon_{1}}\right),
$$

following three steps:

- The energy ratio is monitored during the calculation by evaluating the resolved energy and the total energy. This ratio is called the observed ratio $r^{o}$.

- This ratio is compared to the ratio used in Eq. (18), which is called the target ratio $r^{t}$.

- The coefficient $C_{\varepsilon_{2}}^{*}$ entering the dissipation equation is replaced by $C_{\varepsilon_{2}}^{*}+\delta C_{\varepsilon_{2}}^{*}$, in order to drive the observed ratio toward the target ratio.

This procedure is rather simple in principle, but requires some criterion to determine the amplitude of the correction $\delta C_{\varepsilon_{2}}^{*}$. Obviously, this correction must vanish when the target is approached. Therefore, an estimate of $\delta C_{\varepsilon_{2}}^{*}$ as a function of $r^{o}$ and $r^{t}$ is necessary. Such an estimate can be obtained by simply considering the modification of the level of SGS energy induced by a modification of the $C_{\varepsilon_{2}}^{*}$ coefficient.

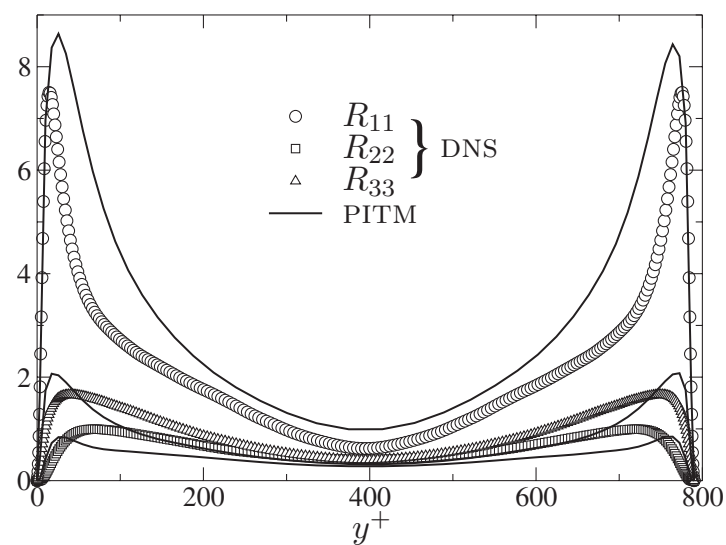

FIG. 11. Influence of the function $f_{\mathrm{SGS}}$ (formulation P-EB with $b=0.5$ ) on the normal components of the Reynolds stress $\left(R_{i j}^{+}=\overline{u_{i}^{\prime} u_{j}^{\prime}}+{\overline{\tau_{i j}}}^{+}\right)$. Reference mesh $\mathbf{M}^{0}$. 

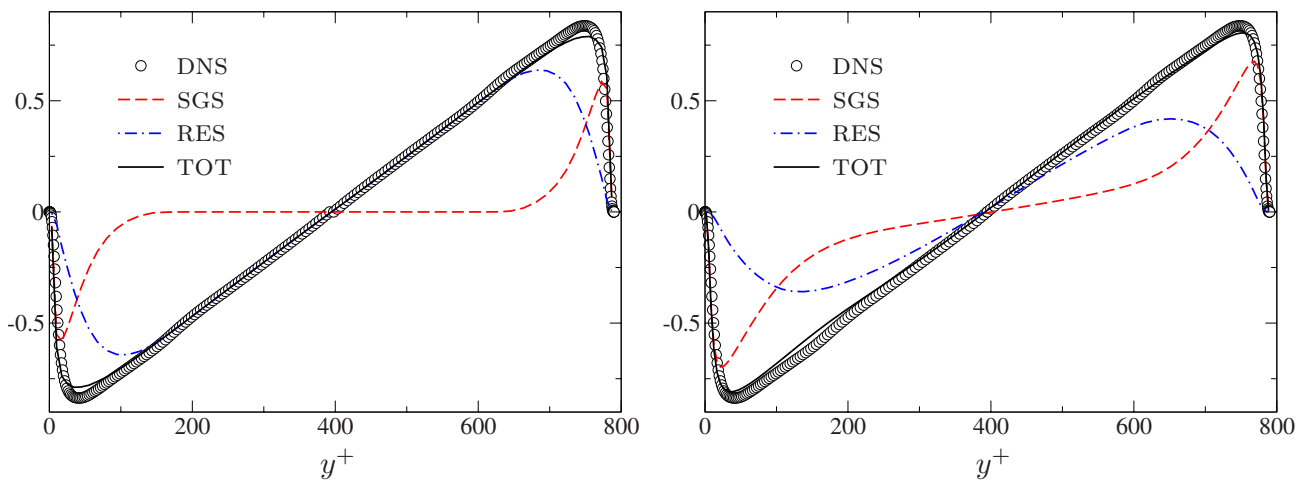

FIG. 12. (Color online) Influence of the function $f_{\mathrm{SGS}}$ (formulation P-EB with $\left.b=0.5\right)$ on the resolved $\left(\mathrm{RES}=\overline{u^{\prime} v^{\prime}}\right)$, modeled $\left(\mathrm{SGS}=\overline{\bar{\tau}_{12}}+\right.$ ), and total $\left(\mathrm{TOT}=R_{12}^{+}=\mathrm{RES}+\mathrm{SGS}\right)$ shear stress. Left: mesh $\mathrm{M}^{0}$. Right: mesh $\mathrm{M}^{-}$.

Indeed, in homogeneous turbulence, it is well known that a system of equation such as Eqs. (15) and (16) tends to a weak equilibrium solution at large times characterized by

$$
\eta_{m}^{2}=\frac{C_{\varepsilon 2}^{*}-1}{C_{\mu}\left(C_{\varepsilon 1}-1\right)},
$$

where $\eta_{m}=S k_{m} / \varepsilon$ and $S$ is the constant mean shear. This equation shows that a small perturbation $\delta C_{\varepsilon_{2}}^{*}$ of the $C_{\varepsilon_{2}}^{*}$ coefficient leads to a perturbation of $\eta_{m}$ at equilibrium given by

$$
2 \frac{\delta \eta_{m}}{\eta_{m}}=\frac{\delta C_{\varepsilon_{2}}^{*}}{C_{\varepsilon_{2}}^{*}-1} .
$$

Now, at the RANS limit $\left(C_{\varepsilon 2}^{*}=C_{\varepsilon 2}\right)$, the equilibrium is characterized by $\eta=S k / \varepsilon$. Therefore, the ratio $r=k_{m} / k$ reads

$$
r=\frac{k_{m}}{k}=\frac{\eta_{m}}{\eta},
$$

such that the perturbation $\delta r$ of the ratio $r$ is

$$
\frac{\delta r}{r}=\frac{\delta \eta_{m}}{\eta_{m}}=\frac{1}{2} \frac{\delta C_{\varepsilon_{2}}^{*}}{C_{\varepsilon_{2}}^{*}-1} .
$$

In order to reach the target ratio $r^{t}$, the desired variation $\delta r$ is $r^{t}-r^{o}$, such that the following relation is obtained:

$$
\delta C_{\varepsilon_{2}}^{*}=\underbrace{2\left(C_{\varepsilon_{2}}^{*}-1\right)}_{\kappa}\left(\frac{r^{t}}{r^{o}}-1\right) .
$$

This relation merely provides an estimate of the dynamic correction to be applied. In practice, the parameter $\aleph$ is considered a constant and adjusted in order to counteract the above-mentioned drift of the computation toward a steady solution during the transient phase. Using this dynamic procedure, the unphysical averaging of the source terms of Eq. (8), temporarily used in previous sections to help sustaining an unsteady resolved field, is avoided. Averaging is only used to evaluate the statistical quantities involved in the model, i.e., the total energy $k$ and the dissipation rate $\varepsilon$, entering the integral length scale in Eq. (57), and the modeled energy $k_{m}$ entering the definition of $r^{o}$. Note that in the present case, averaging is performed in time and in homogeneous directions.

It is worth emphasizing that, although the dynamic procedure is, in practice, an important ingredient of the model, it does not modify its rationale described in previous sections. Indeed, when the permanent state is reached during the computation, the observed ratio $r^{o}$ approaches the target ratio $r^{t}$, such that the dynamic correction equation (71) becomes small. Thus, the parameter driving the partition among resolved and modeled energy remains $r^{t}$, evaluated by Eq. (57).

The model resulting from all the ingredients selected in Sec. IV is finally applied using the dynamic procedure. The final model thus consists of the filtered momentum equation (7), the SGS tensor transport equation (8), using the ellipticblending model described in Sec. III B, the dissipation rate transport equation (44), and the elliptic relaxation equation (39) for the blending coefficient $\alpha$. According to the conclusions drawn from Sec. IV and from the present section,

- The target energy ratio $r^{t}$ is provided by Eq. (57), with $p=2$ and $\beta_{0}=0.20$. In this relation, the integral length scale is initialized by the result of a RANS computation, and updated during the computation, after a transient phase.

- This ratio $r^{t}$ is used in Eq. (18) to evaluate $C_{\varepsilon_{2}}^{*}$, and the dynamic correction $\delta C_{\varepsilon_{2}}^{*}$ is applied.

- The length scale of the blocking effect in Eq. (39) is modeled by Eq. (62).

- The modification $f_{\mathrm{SGS}}$ of the slow term described in Sec. IV D is not used.

In this section, dedicated to the final validation of the method, a third mesh, finer than the reference mesh $\mathrm{M}^{0}$, is introduced, in order to investigate the behavior of the model when LES is approached. This mesh, denoted by $\mathrm{M}^{+}$, is obtained by refining the reference mesh $\mathrm{M}^{0}$ by a factor of 1.5 in all directions. The results for the three meshes $\mathbf{M}^{-}, \mathbf{M}^{0}$, and $\mathrm{M}^{+}$are shown in Figs. 13-17. In Figs. 13 and 14, the shear stress and the turbulent energy, respectively, are compared 

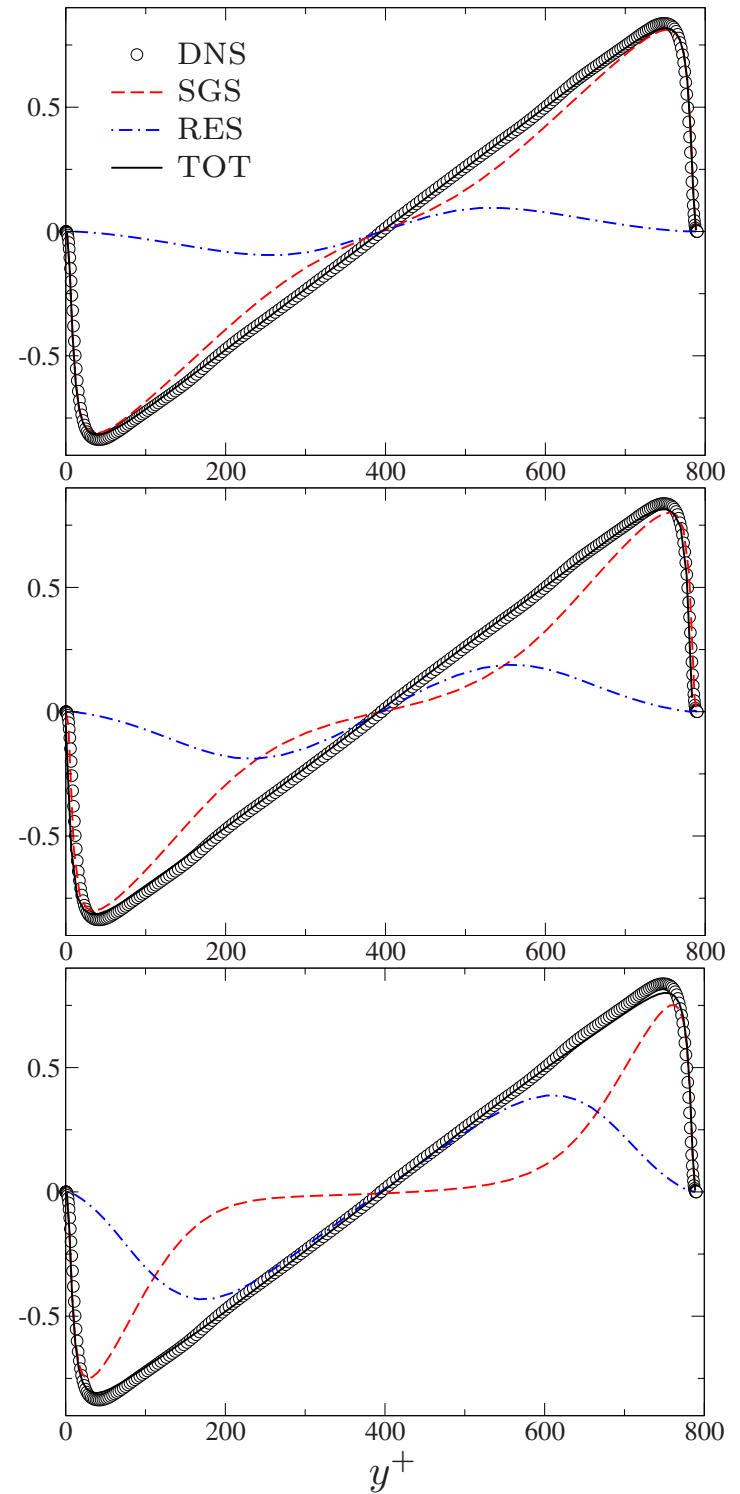

FIG. 13. (Color online) Dynamic approach. Profile of resolved (RES $\left.=\overline{u^{\prime} v^{\prime}}\right)$, modeled $\left(\mathrm{SGS}={\overline{\tau_{12}}}^{+}\right)$, and total $(\mathrm{TOT}=\mathrm{RES}+\mathrm{SGS})$ shear stress. From top to bottom: mesh $\mathrm{M}^{-}$; mesh $\mathrm{M}^{0}$; mesh $\mathrm{M}^{+}$.

against the DNS data, together with their two contributions (resolved and modeled parts). It is noted, compared to previous sections, in which the artificial averaging of the source terms was used, that the computations using the dynamic procedure do not exhibit a pseudo-DNS behavior in the center of the channel because the partition between resolved and modeled energy is better controlled. In Fig. 14, a remarkable feature of the model can be observed: when the mesh is progressively refined, the partition of turbulent kinetic energy among resolved and modeled scales is drastically modified, but the total of the two contributions remains almost constant.

Figure 16 shows the evolution of the ratio modeled energy/total energy measured in the solution (observed), in comparison with the target ratios, for the three meshes. It can be seen that mesh refinement drives the computation toward the LES mode in an increasing portion of the channel. With the finest mesh $\mathrm{M}^{+}$, if Pope's criterion is retained, ${ }^{58}$ i.e., if a
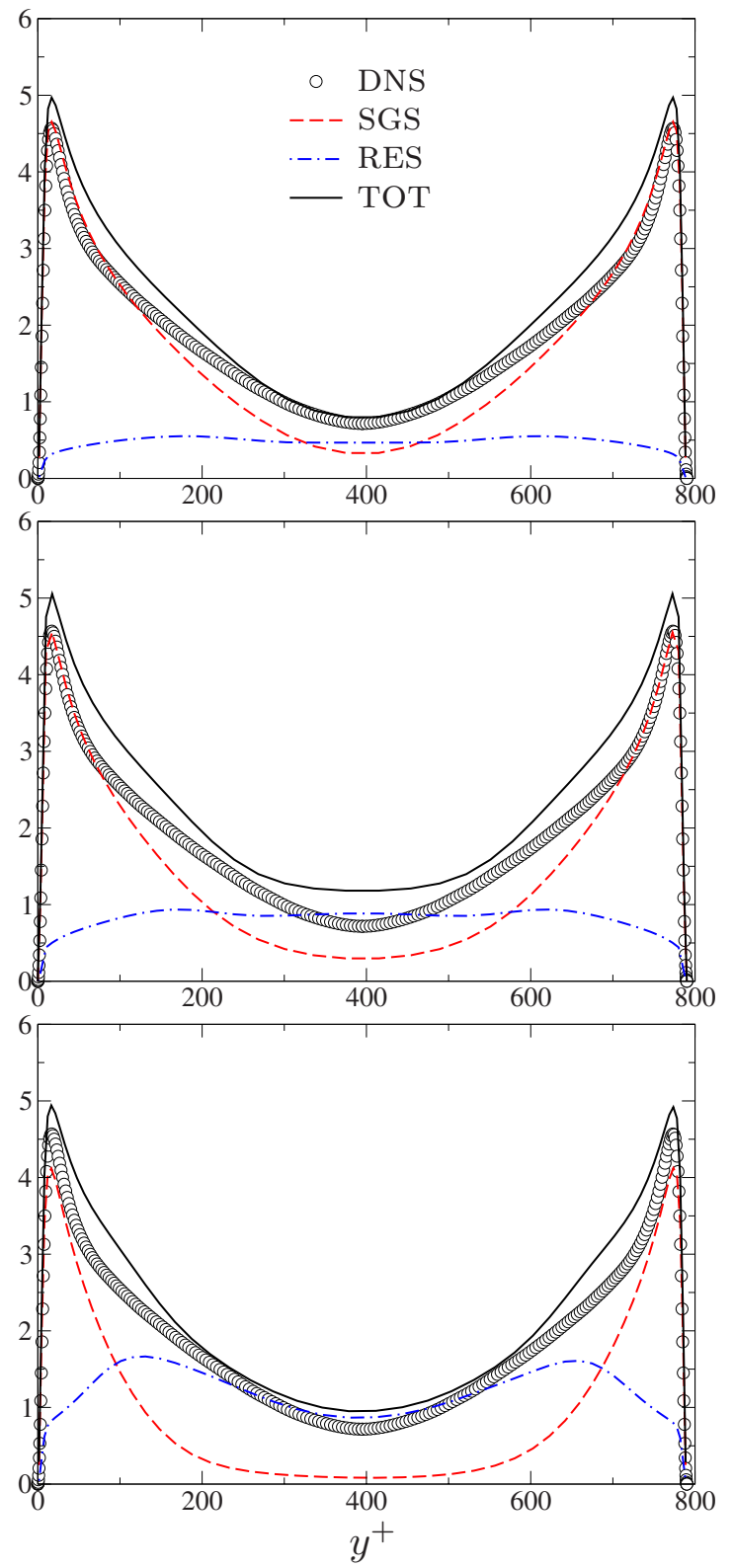

FIG. 14. (Color online) Same figure as Fig. 13 for the turbulent kinetic energy.

LES is characterized by $80 \%$ of resolved energy, the model is in LES mode in about half of the channel. Note that since the RANS mode is enforced in the near-wall region by Eq. (57), further refining the mesh in this region would not lead to performing a full LES. With the other two meshes, the computation can only be considered a VLES.

Moreover, this figure illustrates the difficulty to control the energy partition, since the target value $r^{t}$ is never exactly obtained, in particular in the central part of the channel, where the difference can reach about $20 \%$ with mesh $\mathrm{M}^{0}$. In the near-wall region, the ratio modeled energy/total energy does not reach 1 , which is due to the unsteadiness generated by structures computed in the outer region.

Figure 15 shows the normal Reynolds stresses obtained with the dynamic approach on the three meshes. It can be seen that these components are more sensitive to mesh refinement than the turbulent energy, i.e., even if the total en- 

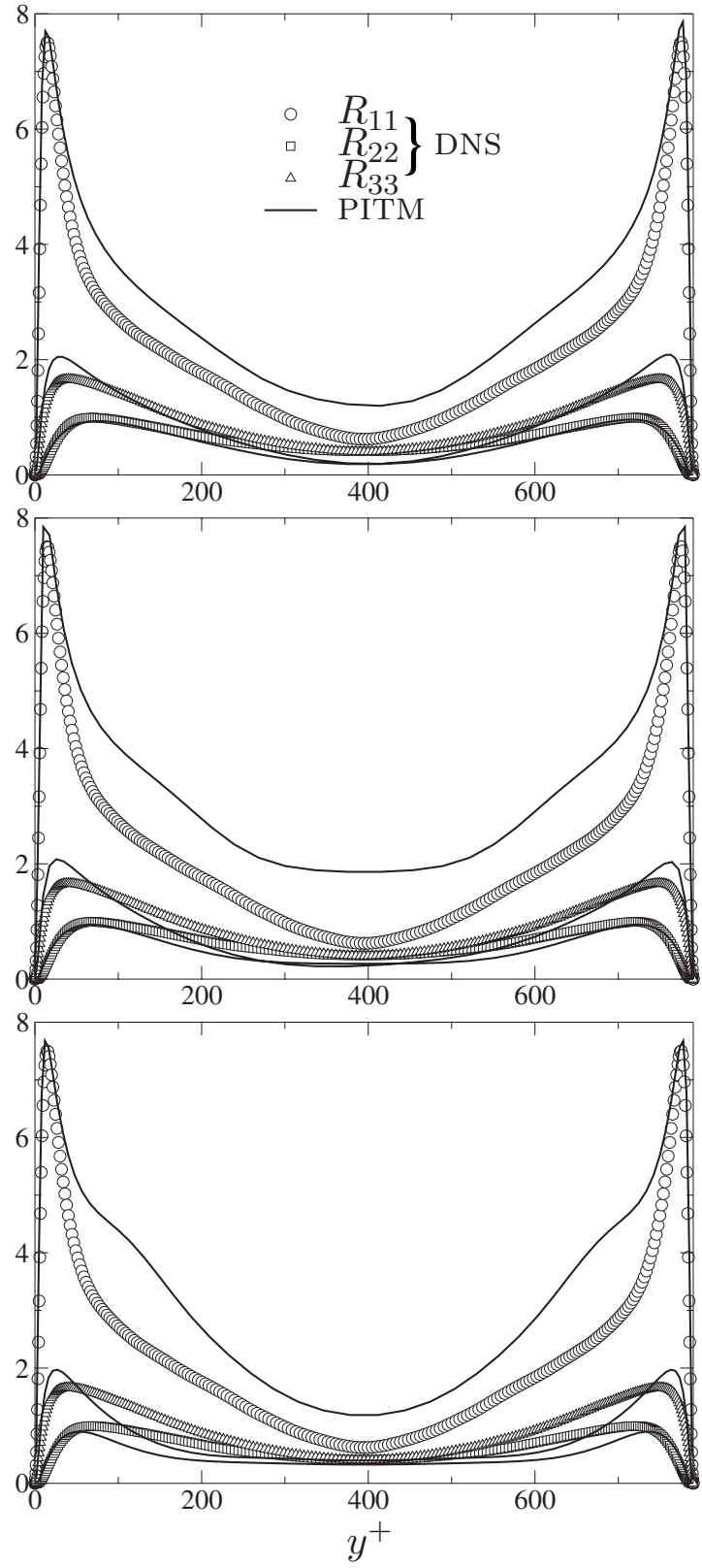

FIG. 15. Dynamic approach. Total $\left(R_{i j}=\overline{u_{i}^{\prime} u_{j}^{\prime}}+\overline{\tau_{i j}}+\right)$ normal Reynolds stresses. From top to bottom: mesh $\mathrm{M}^{-}$; mesh $\mathrm{M}^{0}$; mesh $\mathrm{M}^{+}$.

ergy is preserved, the anisotropy is modified. The streamwise component is overestimated with the three meshes and, in particular, with the finest mesh $\mathrm{M}^{+}$, in the region around $y^{+}=100$.

However, this misprediction does not affect the shear stress (Fig. 13) and the mean velocity, which is shown in Fig. 17, for the three meshes. Comparison is done with DNS and a RANS computation with the EB-RSM. Even though the reproduction of the buffer and logarithmic regions is not as satisfactory as with the RANS model, the mean velocity profile is correctly reproduced. The noticeable independence of the mesh, observed in this figure as well as in Fig. 14, illustrates the fact that the goal of the present work, which is to develop a model able to provide acceptable results whatever the mesh, is achieved.

These results are very similar to those obtained by

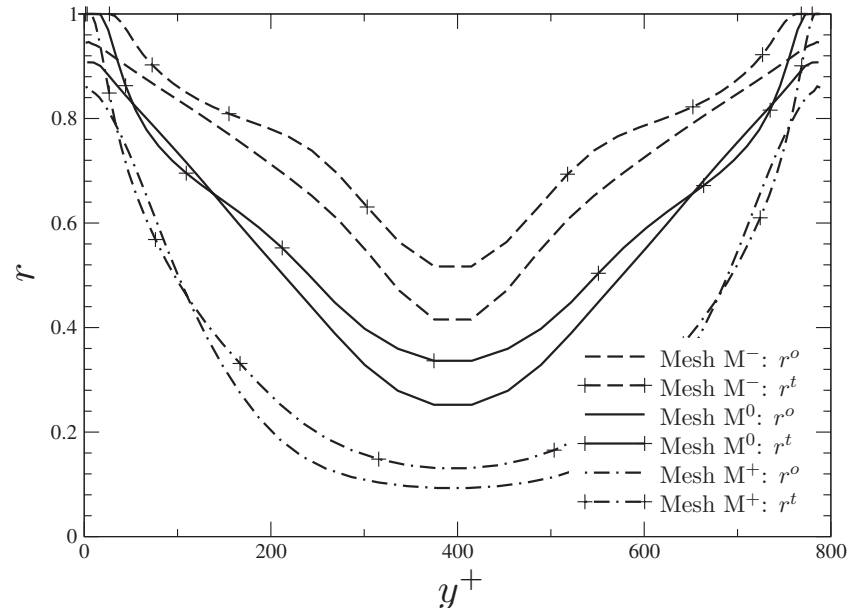

FIG. 16. Observed and target ratios modeled/total kinetic energy.

Chaouat and Schiestel ${ }^{14,44}$ using a different model for the pressure-strain correlation and damping functions in the near-wall region. The aim of the present work was not to improve the results of these authors in channel flows, which were already satisfactory, but to provide a model free of damping functions. Similar to what has been shown during the past two decades in RANS, it is expected that a near-wall model based on a theoretical basis, such as the elliptic relaxation approach, will be of more general applicability than a model based on empirical damping functions.

It must be pointed out that on a given mesh, solving the eight differential equations used to model the subfilter stresses increases the computational cost by a factor of about 1.6 compared to a Smagorinsky model. This higher cost is by far compensated by the possibility of coarsening the mesh. For example, in the case of a channel flow with fluid injections by the porous walls, Chaouat and Schiestel ${ }^{14}$ have obtained the same results as a classical LES with a Smagorinsky model, but with a mesh seven times coarser with the PITM approach. Actually, in the finite volume code used in the present work, most of the computational cost is due to the resolution of the velocity/pressure system. Therefore, the

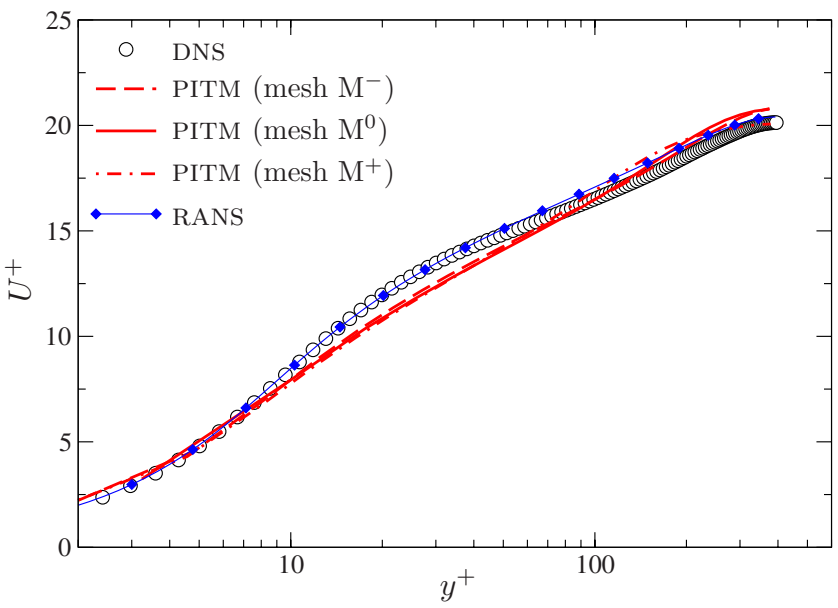

FIG. 17. (Color online) Mean velocity profiles. 
resolution of the additional equations for the subgrid stresses, the dissipation rate and the elliptic-blending function is affordable.

\section{CONCLUSIONS}

A new seamless hybrid RANS-LES model has been developed, based on transport equations for the subgrid stresses, and the elliptic-blending method to account for the nonlocal kinematic blocking effect of the wall. The derivation is made in the framework of the PITM strategy proposed by Schiestel and Dejoan ${ }^{13}$ and Chaouat and Schiestel. ${ }^{14}$ The purpose of such a model is to obtain the unsteady characteristics of the flow at a cost lower than LES, by going to a RANS computation in the near-wall regions, and also by making the use of coarse meshes possible (compared to LES meshes). However, when the grid is fine enough such that the cutoff wavenumber is in the inertial range of the energy spectrum, it has been shown that the PITM is equivalent to a Smagorinsky-type model, and therefore compatibility with the LES limit is guaranteed. When the grid is coarse, the cutoff can be located in the productive zone of the spectrum, and thus, the complex production and redistribution mechanisms must be reproduced, due to high anisotropies. A second-moment closure is believed to give a better representation of these physical processes, in comparison to eddyviscosity models, especially in an unsteady context (e.g., Carpy and Manceau ${ }^{22}$ ). For this purpose, the ellipticblending hybrid model is used and based on transport equations for the SGS tensor and the dissipation rate. It is shown that the elliptic relaxation strategy of Durbin ${ }^{26}$ is valid in a RANS (steady) as well as a LES context (unsteady). The wall-blocking effect is reproduced by using an additional elliptic relaxation equation for the blending function $\alpha$, which drives the transition of the SGS pressure term from a nearwall behavior to a quasihomogeneous behavior. A new form of the parameter $r$, which provides the model with the target ratio modeled/total kinetic energy, is proposed to better control the RANS-LES transition in the near-wall regions, and is calibrated in the channel flow at $\operatorname{Re}_{\tau}=395$. A new formulation of the correlation length scale for the elliptic relaxation equation is also proposed, in order to account for the fact that the kinematic blocking effect must be imposed only on the SGSs. Finally, a dynamic correction of the variable coefficient $C_{\varepsilon_{2}}^{*}$ in the dissipation rate equation is proposed in order to ensure that the ratio modeled/total kinetic energy observed in the results is the same as the target ratio imposed in the equations of the model. Comparisons with the DNS data ${ }^{38}$ show that the results in channel flow are very encouraging in terms of turbulence statistics, and are remarkably independent of the mesh. Near the wall, the SGS part is dominant (RANS mode) and decreases toward the center of the channel, where the resolved part in turn becomes dominant (LES mode). As expected, a modification of the mesh modifies the partition between modeled and resolved energy, but the total turbulent energy remains nearly constant. Although the results are very encouraging, further validation of the model in more complex flows is necessary.

\section{ACKNOWLEDGMENTS}

The first author would like to dedicate this work to his beloved father Dr. M. M. Fadai-Ghotbi, who inspired his interest in science. This work was granted access to the HPC resources of IDRIS under Grant No. 2010-020912 made by GENCI (Grand Equipement National de Calcul Intensif).

${ }^{1}$ P. Sagaut, S. Deck, and M. Terracol, Multiscale and Multiresolution Approaches in Turbulence (Imperial College Press, London, 2006).

${ }^{2}$ C. G. Speziale, "Turbulence modeling for time-dependent RANS and VLES: A review," AIAA J. 36, 173 (1998).

${ }^{3}$ P. Batten, U. Goldberg, and S. Chakravarthy, "Interfacing statistical turbulence closures with large-eddy simulation," AIAA J. 42, 485 (2004).

${ }^{4}$ P. R. Spalart, "Strategies for turbulence modeling and simulations," Int. J. Heat Fluid Flow 21, 252 (2000).

${ }^{5}$ W. C. Lasher and D. B. Taulbee, "On the computation of turbulent backstep flow," Int. J. Heat Fluid Flow 13, 30 (1992).

${ }^{6}$ G. Bosch and W. Rodi, "Simulation of vortex shedding past a square cylinder with different turbulence models," Int. J. Numer. Methods Fluids 28, 601 (1998).

${ }^{7}$ P. Reynier and H. Ha Minh, "Numerical prediction of unsteady compressible turbulent coaxial jets," Computers and Fluids, 27, 239 (1998).

${ }^{8}$ G. Iaccarino, A. Ooi, P. A. Durbin, and M. Behnia, "Reynolds averaged simulation of unsteady separated flow," Int. J. Heat Fluid Flow 24, 147 (2003).

${ }^{9}$ J. Paik, L. Ge, and F. Sotiropoulos, "Toward the simulation of complex 3D shear flows using unsteady statistical turbulence models," Int. J. Heat Fluid Flow 25, 513 (2004).

${ }^{10}$ Y. Hoarau, R. Perrin, M. Braza, D. Ruiz, and G. Tzabiras, "Advances in turbulence modelling for unsteady flows-IMFT," in FLOMANIA-A European Initiative on Flow Physics Modelling, Notes on Numerical Fluid Mechanics and Multidisciplinary Design Vol. 94 (Springer, Berlin, 2006), pp. $85-88$.

${ }^{11}$ F. R. Menter and Y. Egorov, "SAS turbulence modeling of technical flows," in Proceedings of the Sixth International ERCOFTAC Workshop on Direct and Large-Eddy Simulation, Poitiers, France, 2005, Vol. 10, pp. 687-694.

${ }^{12}$ S. S. Girimaji, "Partially-averaged Navier-Stokes model for turbulence: A Reynolds-averaged Navier-Stokes to direct numerical simulation bridging method," J. Appl. Mech. 73, 413 (2006).

${ }^{13}$ R. Schiestel and A. Dejoan, "Towards a new partially integrated transport model for coarse grid and unsteady turbulent flow simulations," Theor. Comput. Fluid Dyn. 18, 443 (2005).

${ }^{14}$ B. Chaouat and R. Schiestel, "A new partially integrated transport model for subgrid-scale stresses and dissipation rate for turbulent developing flows," Phys. Fluids 17, 065106 (2005).

${ }^{15}$ M. Germano, "Properties of the hybrid RANS/LES filter," Theor. Comput. Fluid Dyn. 17, 225 (2004).

${ }^{16}$ F. Hamba, "Log-layer mismatch and commutation error in hybrid RANS/ LES simulation of channel flow," Int. J. Heat Fluid Flow 30, 20 (2009).

${ }^{17}$ M. Sánchez-Rocha and S. Menon, "The compressible hybrid RANS/LES formulation using an additive operator," J. Comput. Phys. 228, 2037 (2009).

${ }^{18}$ J. Fröhlich and D. von Terzi, "Hybrid LES/RANS methods for the simulation of turbulent flows," Prog. Aerosp. Sci. 44, 349 (2008).

${ }^{19}$ T. B. Gatski, C. L. Rumsey, and R. Manceau, "Current trends in modelling research for turbulent aerodynamic flows," Philos. Trans. R. Soc. London, Ser. A 365, 2389 (2007).

${ }^{20}$ A. Fadai-Ghotbi, Ch. Friess, R. Manceau, T. Gatski, and J. Borée, "Temporal filtering: A consistent formalism for seamless hybrid RANS-LES modeling in inhomogeneous turbulence," Int. J. Heat Fluid Flow (in press).

${ }^{21}$ I. Hadžić, K. Hanjalić, and D. Laurence, "Modeling the response of turbulence subjected to cyclic irrotational strain," Phys. Fluids 13, 1739 (2001).

${ }^{22}$ S. Carpy and R. Manceau, "Turbulence modeling of statistically periodic flows: Synthetic jet into quiescent air," Int. J. Heat Fluid Flow 27, 756 (2006).

${ }^{23}$ J. W. Deardorff, "A numerical study of three-dimensional turbulent channel flow at large Reynolds number," J. Fluid Mech. 41, 453 (1970). 
${ }^{24}$ U. Schumann, "Subgrid scale model for finite difference simulations of turbulent flows in plane channels and annuli," J. Comput. Phys. 18, 376 (1975).

${ }^{25}$ J. B. Perot and J. Gadebusch, "A stress transport equation model for simulating turbulence at any mesh resolution," Theor. Comput. Fluid Dyn. 23, 271 (2009)

${ }^{26}$ P. A. Durbin, "A Reynolds stress model for near-wall turbulence," J. Fluid Mech. 249, 465 (1993).

${ }^{27}$ R. Manceau and K. Hanjalić, "Elliptic blending model: A new near-wall Reynolds-stress turbulence closure," Phys. Fluids 14, 744 (2002).

${ }^{28} \mathrm{R}$. Manceau, "An improved version of the elliptic blending model. Application to non-rotating and rotating channel flows," in Proceedings of the Fourth International Symposium on Turbulence Shear Flow Phenomena, Williamsburg, VA, 2005.

${ }^{29}$ C. G. Speziale, S. Sarkar, and T. B. Gatski, "Modeling the pressure-strain correlation of turbulence: An invariant dynamical system approach," J. Fluid Mech. 227, 245 (1991).

${ }^{30}$ J. K. Shin, K. H. Chun, and Y. D. Choi, "Refinement of a second moment closure by the elliptic blending equation and its application to turbulent rotating channel flows," J. Turbul. 4, 30 (2003).

${ }^{31}$ L. Thielen, K. Hanjalić, H. Jonker, and R. Manceau, "Predictions of flow and heat transfer in multiple impinging jets with an elliptic-blending second-moment closure," Int. J. Heat Mass Transfer 48, 1583 (2005).

${ }^{32}$ D. Borello, K. Hanjalić, and F. Rispoli, "Prediction of cascade flows with innovative second-moment closure," J. Fluids Eng. 127, 1059 (2005).

${ }^{33}$ S. H. Han, Y. D. Choi, J. K. Shin, Y. C. Kim, and M. S. Kim, "Turbulent heat transfer of supercritical carbon dioxide in square cross-sectional duct flow," J. Mech. Sci. Technol. 22, 2563 (2008).

${ }^{34}$ S.-K. Choi and S.-O. Kim, "Computation of a turbulent natural convection in a rectangular cavity with the elliptic-blending second-moment closure," Int. Commun. Heat Mass Transfer 33, 1217 (2006).

${ }^{35}$ V. Viti, G. Huang, and P. Bradshaw, "Numerical study of stress-transport turbulence models: Implementation and validation issues," Computers and Fluids 36, 1373 (2007).

${ }^{36}$ J. K. Shin, J. S. An, Y. D. Choi, Y. C. Kim, and M. S. Kim, "Elliptic relaxation second moment closure for the turbulent heat fluxes," J. Turbul. 9, 3 (2008).

${ }^{37}$ M. Germano, "Turbulence: The filtering approach,” J. Fluid Mech. 238, 325 (1992).

${ }^{38}$ R. D. Moser, J. Kim, and N. N. Mansour, "Direct numerical simulation of turbulent channel flow up to $R e_{\tau}=590$," Phys. Fluids 11, 943 (1999).

${ }^{39}$ B. Chaouat and R. Schiestel, "From single-scale turbulence models to multiple-scale models by Fourier transform," Theor. Comput. Fluid Dyn. 21, 201 (2007).

${ }^{40} \mathrm{R}$. Schiestel, "Sur le concept d'échelles multiples en modélisation des écoulements turbulents (partie I),” J. Mec. Theor. Appl. 2, 417 (1983).

${ }^{41} \mathrm{R}$. Schiestel, "Sur le concept d'échelles multiples en modélisation des écoulements turbulents (partie II)," J. Mec. Theor. Appl. 2, 601 (1983).

${ }^{42} \mathrm{R}$. Schiestel, "Multiple-time-scale modeling of turbulent flows in onepoint closures," Phys. Fluids 30, 722 (1987).

${ }^{43}$ C. Bailly and G. Comte-Bellot, Turbulence (CNRS Éditions, Paris, 2003).

${ }^{44}$ B. Chaouat and R. Schiestel, "Progress in subgrid-scale transport modeling for continuous hybrid non-zonal RANS/LES simulations," Int. J. Heat Fluid Flow 30, 602 (2009).

${ }^{45}$ A. J. Revell, S. Benhamadouche, T. Craft, and D. Laurence, "A stress- strain lag eddy viscosity model for unsteady mean flow," Int. J. Heat Fluid Flow 27, 821 (2006).

${ }^{46}$ S. Aubrun, P. L. Kao, and H. C. Boisson, "Experimental coherent structures extraction and numerical semi-deterministic modeling in the turbulent flow behind a backward-facing step," Exp. Therm. Fluid Sci. 22, 93 (2000).

${ }^{47}$ P. A. Durbin, "Near-wall turbulence closure modeling without damping functions," Theor. Comput. Fluid Dyn. 3, 1 (1991).

${ }^{48}$ R. Manceau, M. Wang, and D. Laurence, "Inhomogeneity and anisotropy effects on the redistribution term in Reynolds-averaged Navier-Stokes modelling," J. Fluid Mech. 438, 307 (2001).

${ }^{49}$ D. Euvrard, Résolution Numérique des Équations aux Dérivées Partielles de la Physique, de la Mécanique et des Sciences de l'Ingénieur. Différences Finies, Éléments Finis, Problèmes en Domaine Non Borné (Masson, Paris, 1994).

${ }^{50}$ V. Wizman, D. Laurence, M. Kanniche, P. Durbin, and A. Demuren, "Modeling near-wall effects in second-moment closures by elliptic relaxation,” Int. J. Heat Fluid Flow 17, 255 (1996).

${ }^{51}$ R. Manceau and K. Hanjalić, "A new form of the elliptic relaxation equation to account for wall effects in RANS modeling," Phys. Fluids 12, 2345 (2000).

${ }^{52}$ R. Manceau, J. R. Carlson, and T. B. Gatski, "A rescaled elliptic relaxation approach: Neutralizing the effect on the log layer," Phys. Fluids 14, 3868 (2002).

${ }^{53}$ P. A. Durbin and C. G. Speziale, "Local anisotropy in strained turbulence at high Reynolds numbers," J. Fluids Eng. 113, 707 (1991).

${ }^{54}$ J. L. Lumley, "Computational modeling of turbulent flows," in Advances in Applied Mechanics (Academic, New York, 1978), Vol. 18, pp. 123-175.

${ }^{55}$ J. C. Rotta, "Statistische Theorie nichthomogener Turbulenz," Z. Phys. 129, 547 (1951)

${ }^{56}$ B. J. Daly and F. H. Harlow, "Transport equations in turbulence," Phys. Fluids 13, 2634 (1970).

${ }^{57}$ J. O. Hinze, Turbulence (McGraw-Hill, New-York, 1975).

${ }^{58}$ S. Pope, Turbulent Flows (Cambridge University Press, New York, 2000).

${ }^{59}$ F. Archambeau, N. Méchitoua, and M. Sakiz, "Code Saturne: A finite volume code for the computation of turbulent incompressible flowsIndustrial applications," electronic edition: http://averoes.math.univparis13.fr/html (2004).

${ }^{60}$ J. H. Ferziger and M. Perić, Computational Methods for Fluid Dynamics (Springer, New York, 1996).

${ }^{61}$ P. R. Spalart, T. Deck, M. L. Shur, M. K. Squires, K. D. Strelets, and A. Travin, "A new version of detached-eddy simulation, resistant to ambiguous grid densities," Theor. Comput. Fluid Dyn. 20, 181 (2006).

${ }^{62} \mathrm{~S}$. Ghosal, "An analysis of numerical errors in large eddy simulations of turbulence," J. Comput. Phys. 125, 187 (1996).

${ }^{63}$ J. C. R. Hunt and J. M. R. Graham, "Free stream turbulence near plane boundaries," J. Fluid Mech. 84, 209 (1978).

${ }^{64}$ B. Perot and P. Moin, "Shear-free turbulent boundary layers. Part 1. Physical insights into near-wall turbulence," J. Fluid Mech. 295, 199 (1995).

${ }^{65} \mathrm{R}$. Schiestel, "Studying turbulence using numerical simulation databases," in Proceedings of the Summer Program of the Center for Turbulence Research, Stanford University, CA, 1987, pp. 95-108.

${ }^{66} \mathrm{R}$. Schiestel, Méthodes de Modélisation et de Simulation des Écoulements Turbulents (Hermes/Lavoisier, Paris, 2006). 